\title{
Modeling and Evaluation of a Ridesharing Matching System from Multi-Stakeholders' Perspective
}

\author{
Md Tawhidur Rahman \\ West Virginia University, mr0086@mix.wvu.edu
}

Follow this and additional works at: https://researchrepository.wvu.edu/etd

Part of the Transportation Engineering Commons

\section{Recommended Citation \\ Rahman, Md Tawhidur, "Modeling and Evaluation of a Ridesharing Matching System from Multi- Stakeholders' Perspective" (2018). Graduate Theses, Dissertations, and Problem Reports. 3727. https://researchrepository.wvu.edu/etd/3727}

This Thesis is protected by copyright and/or related rights. It has been brought to you by the The Research Repository @ WVU with permission from the rights-holder(s). You are free to use this Thesis in any way that is permitted by the copyright and related rights legislation that applies to your use. For other uses you must obtain permission from the rights-holder(s) directly, unless additional rights are indicated by a Creative Commons license in the record and/ or on the work itself. This Thesis has been accepted for inclusion in WVU Graduate Theses, Dissertations, and Problem Reports collection by an authorized administrator of The Research Repository @ WVU. For more information, please contact researchrepository@mail.wvu.edu. 


\title{
Modeling and Evaluation of a Ridesharing Matching System from Multi-Stakeholders' Perspective
}

\author{
Md Tawhidur Rahman
}

Thesis submitted to the

Benjamin M. Statler College of Engineering and Mineral Resources at West Virginia University

In partial fulfillment of the requirements for the degree of

Master of Science in

Civil Engineering

Kakan Dey, Ph.D., Chair

David R. Martinelli, Ph.D.

Alan R. McKendall, Ph.D.

Department of Civil and Environmental Engineering

\author{
Morgantown, West Virginia \\ 2018
}

Keywords:

Ridesharing, Matching, Optimization, VMT, Waiting Time, Detour, Profit Copyright 2018, Md Tawhidur Rahman 


\title{
ABSTRACT \\ Modeling and Evaluation of a Ridesharing Matching System from Multi- Stakeholders' Perspective
}

\author{
Md Tawhidur Rahman
}

With increasing travel demand and mobility service quality expectations, demand responsive innovative services continue to emerge. Ridesharing is an established, yet evolving, mobility option that can provide more customized, reliable shared service without any new investment in the transportation infrastructure. To maximize the benefits of ridesharing service, efficient matching and distribution of riders among available drivers can provide a reliable mobility option under most operating conditions. Service efficiency of ridesharing depends on the system performance (e.g., trip travel time, trip delay, trip distance, detour distance, and trip satisfaction) acceptable to diverse mobility stakeholders (e.g., riders, drivers, ridesharing operators, and transportation agencies). This research modeled the performance of a ridesharing service system considering four objectives: (i) minimization of system-wide passengers' waiting time, (ii) minimization of system-wide vehicle miles travelled (VMT), (iii) minimization of system-wide detour distance, and (iv) maximization of system-wide drivers' profit. Tradeoff evaluation of objectives revealed that system-wide VMT minimization objective performed best with least sacrifices on the other three objectives from their respective best performance level based on set of routes generated in this study. On the other hand, system-wide drivers' profit maximization objective provided highest monetary incentives for drivers and riders in terms of maximizing profit and saving travel cost respectively. System-wide minimization of detour distance was found to be least flexible in providing shared rides. The findings of this research provide useful insights on ridesharing system modeling and performance evaluation, and can be used in developing and implementing ridesharing service considering multiple stakeholders' concerns. 


\section{ACKNOWLEDGEMENTS}

Firstly, I would like to express my gratitude to my supervisor and committee chair, Dr. Kakan Dey, who has constantly given me inspirations and effective suggestions in regard to research. Without his tireless guidance and co-operation, this thesis would not have been possible.

I would also like to thank other committee members Dr. David R. Martinelli and Dr. Alan R. McKendall for providing me utmost advice, support and encouragement.

I would like to gratefully acknowledge the opportunity West Virginia University have offered me to pursue my MSc program with highly motivated academic and research environment.

Finally, I would like to thank my wife and parents to share the whole journey with me and support me during challenging times faced throughout my academic and research work. 


\section{TABLE OF CONTENTS}

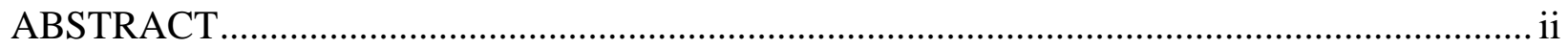

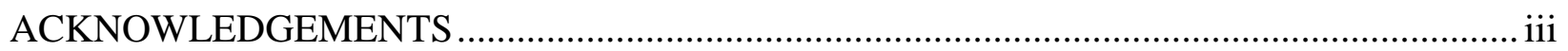

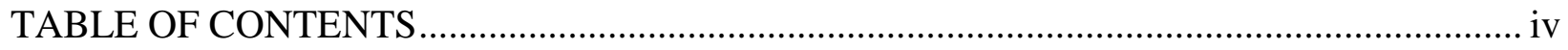

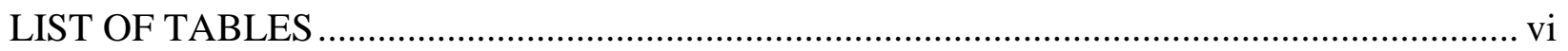

LIST OF FIGURES …........................................................................................... vii

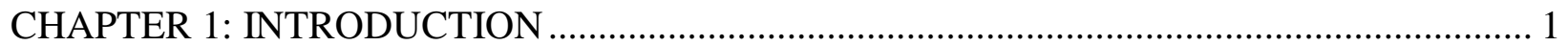

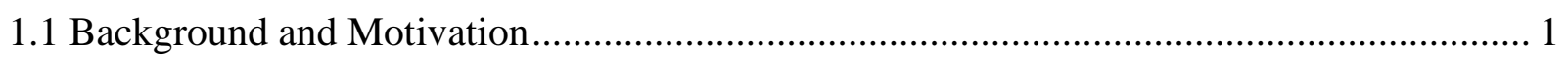

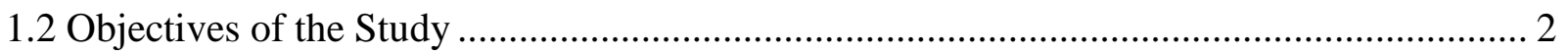

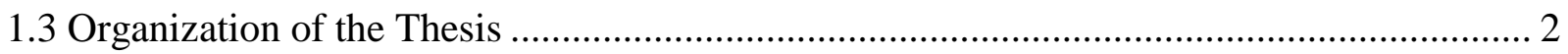

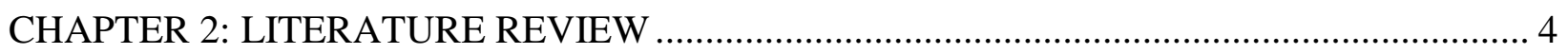

CHAPTER 3: DRIVERS AND RIDERS MATCHING OPTIMIZATION MODELS................ 9

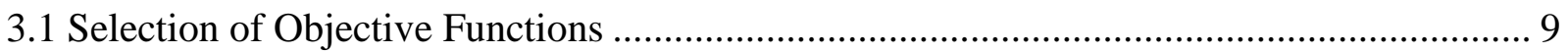

3.2 Model Parameters and Decision Variables ............................................................. 10

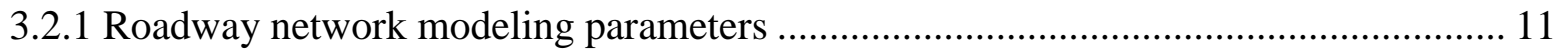

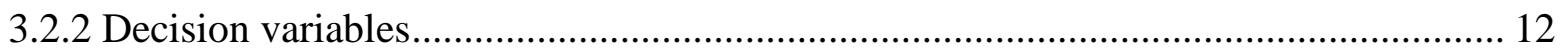

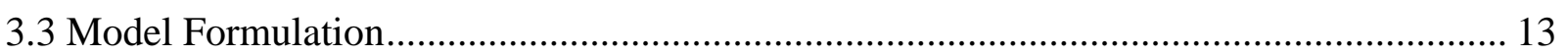

3.3.1 Objective \# 1: Minimization of system-wide waiting time of passengers .................. 13

3.3.2 Objective \# 2: Minimization of system-wide vehicle miles traveled (VMT).............. 14

3.3.3 Objective \# 3: Minimization of system-wide detour distance .................................. 14

3.3.4 Objective \# 4: Maximization of system-wide drivers' profit ................................... 15

CHAPTER 4: ANALYSIS AND RESULTS .................................................................. 16

4.1 Impact of Ridesharing Matching Objectives on Travel Parameters ............................... 18

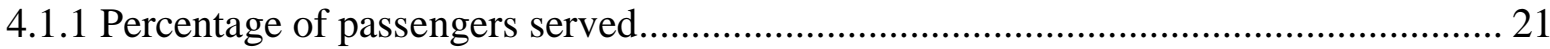

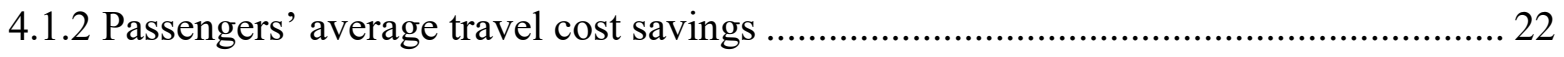

4.1.3 Performance of objectives in terms of ride time index and waiting time index .......... 23

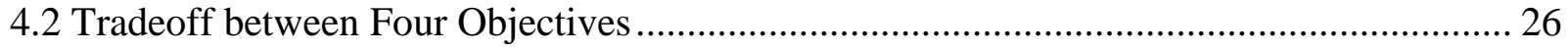

4.2.1 Minimization of system-wide passengers' waiting time as the matching objective .... 28

4.2.2 Minimization of system-wide VMT as the matching objective .............................. 29

4.2.3 Minimization of system-wide detour distance as the matching objective ................... 29 
4.2.4 Maximization of system-wide drivers' profit as the matching objective ................... 30 CHAPTER 5: CONCLUSIONS AND FUTURE RESEARCH DIRECTIONS ........................ 31

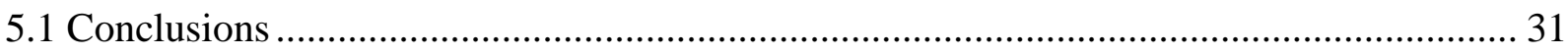

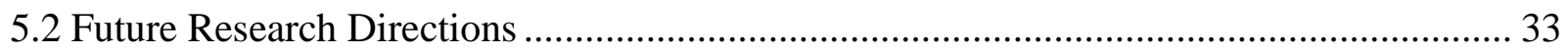

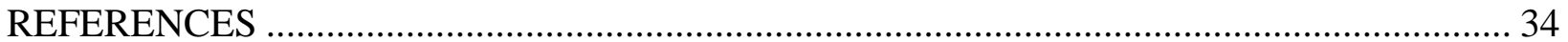




\section{LIST OF TABLES}

Table 1 Information Required from Drivers and Passengers...................... 11

Table 2 Model Parameters....................................................... 11

Table 3 Ridesharing System Parameters Assumed during Matching Objectives' 17 Performance Evaluation 


\section{LIST OF FIGURES}

Figure 1 Hypothetical Grid Network, Drivers' O-D and Passenger Requests' Pick-up and Dropoff Locations Used in Drivers' and Passengers' Matching Optimization 16

Figure 2 Comparison of Four Optimization Objectives' Performance in terms of System-wide Passengers' Waiting Time

Figure 3 Comparison of Four Optimization Objectives' Performance in terms of System-wide VMT.

Figure 4 Comparison of Four Optimization Objectives' Performance in terms of System-wide

Detour Distance

Figure 5 Comparison of Four Optimization Objectives' Performance in terms of System-wide

Drivers' Profit 21

Figure 6 Percentage of Passengers Served through Optimization of Different Objectives ......... 22

Figure 7 Average Travel Cost Saved Per Passenger for Four Objectives .................................... 23

Figure 8 Waiting Time Index of Different Optimization Objectives........................................... 25

Figure 9 Ride Time Index of Different Optimization Objectives .............................................. 25

Figure 10 Tradeoff in terms of Three Objectives When Drivers' and Passengers' Matching Was

Optimized for System-wide Passengers' Waiting Time …………………………………........... 26

Figure 11 Tradeoff in terms of Three Objectives When Drivers' and Passengers' Matching Was

Optimized for System-wide VMT

Figure 12 Tradeoff in terms of Three Objectives When Drivers' and Passengers' Matching Was

Optimized for System-wide Detour Distance.

Figure 13 Tradeoff in terms of Three Objectives When Drivers' and Passengers' Matching Was Optimized for System-wide Drivers' Profit. 28 


\section{CHAPTER 1: INTRODUCTION}

\subsection{Background and Motivation}

During early days of mass automobile use, U.S. transportation policies were focused on accommodating traffic growth by constructing new facilities (Giuliano and Wachs, 1992). Since 1980, policy makers have applied some level of transportation demand management (TDM) to better utilize limited transportation infrastructure and reduce roadway congestion (Hanks, James and Lomax, 1991). TDM are actions that influence travel behavior and promote alternate mobility options such as High Occupancy Vehicle (HOV) lanes (Meyer M.D., 1999). According to 2015 Urban Mobility Scorecard, travelers lost 7 billion hours and wasted 3 billion gallons of fuel due to traffic congestion in the US ("Traffic gridlock," 2015). Due to the lack of physical capacity improvements in the wake of substantial increases in travel demand, many cities in the US and elsewhere experience daily severe recurrent congestion in peak hours at significant economic cost. One of the most important factors related to traffic congestion in the US is reliance on personal vehicles, and single occupancy vehicles (SOVs) which represents more than 76 percent of commuting trips (“Are we reaching," 2015). To support ever-increasing travel demand without significant investment in transportation system capacity improvement, it is important to encourage and promote deployment of emerging mobility services such as shared mobility, which has the potential to substantially increase vehicle occupancy and reduce traffic congestion.

As one of the emerging and innovative passenger transportation services, shared mobility enables riders to minimize dependency on personal vehicles. Ridesharing, a form of shared mobility service, has been growing in popularity and can reduce emissions, fuel consumption, system level VMT, and most importantly, traffic congestion (Jung et al., 2013). According to the United States Civil Defense, ridesharing was first introduced during World War II to conserve rubber for military uses (Cohen and Kietzmann, 2014). Modern-day shared mobility services, enabled by information technology (i.e., mobile app), face several operational challenges such as efficient driver and passenger matching, maintaining acceptable service reliability and flexibility, integration with multimodal options, and multi-institutional collaboration (Amey et al., 2011). Several studies have explored optimization-based matching algorithms considering single objective and multiple objectives to evaluate the ridesharing service performance. Though the objective of ridesharing service varies among stakeholders (e.g., government transportation 
agencies, matching agency, drivers, and riders), past studies have not focused on evaluating the relative performance of each objective used in matching optimization from different stakeholders' perspective. For example, riders prefer minimum waiting time, minimum travel time, and minimum trip cost. On the other hand, drivers prefer maximum profit with minimum detour, while operators prefer maximum profit and maximum matching, and transportation agencies prefer to reduce congestion, air pollution and establish ridesharing as a sustainable mode. The main goal of this research is to investigate how optimization of one objective influences the other objectives to understand the consequence of each individual matching objective on system performance and mobility policy development.

\subsection{Objectives of the Study}

In ridesharing service platforms, efficient matching of drivers and riders is one of the major challenges and is the main focus of this study. Matching efficiency depends on many users and ridesharing system characteristics such as number of available drivers and ride requests, spatial and temporal distribution of drivers and riders, pricing mechanism, request handling mechanism (i.e., static or dynamic), and dependency on other modes. Besides, these transportation system characteristics (e.g., travel time, congestion level, and available routes) also influence matching efficiency in ridesharing significantly. The specific objectives of this study are to:

i. develop model for a ridesharing matching system considering multiple stakeholders' interest,

ii. evaluate the performance of ridesharing matching model, and

iii. investigate the tradeoff associated with adoption of a particular objective with respect to other objectives.

\subsection{Organization of the Thesis}

This manuscript is organized in five chapters. The first chapter provides an introduction on background, motivation and objectives of the study. In chapter 2, related studies on shared ride matching are reviewed and contribution of this research is identified. Chapter 3 focuses on presenting the ridesharing modeling framework, justification of selected objectives, and formulation of matching objective models. Chapter 4 presents analysis and results of ridesharing matching model using a hypothetical roadway network and ridesharing demand scenario. This 
chapter also presents tradeoff analysis associated with the adoption of each matching objective. Chapter 5 summarizes the conclusions of this research along with future research directions. 


\section{CHAPTER 2: LITERATURE REVIEW}

The desire for safe, convenient trip-making with low waiting times, origin-destination flexibility, service reliability, comfort, and privacy have been the major influential factors of using personal vehicles (Beirao and Cabral, 2007). Reliance on personal vehicles is responsible for more than $90 \%$ of VMT and a very low vehicle occupancy rate, which lead to traffic congestion, huge dedicated parking facilities at activity centers (e.g., business districts, shopping mall), higher per person travel cost and emissions. Emerging ridesharing services can reduce the number of singleoccupant vehicles by accommodating the same number of travelers in lesser number of vehicles. Besides that, ridesharing services improve air quality and increase mobility options. But these benefits are gained through sacrifice in conveniences offered by personal vehicles. Moreover, expected benefits of ridesharing vary among stakeholders. For example, government agencies view implementing ridesharing as a mode of serving peoples' transportation need with no/limited investment in infrastructures. On the other hand, ridesharing users focus on reducing their trip travel time and cost. To establish ridesharing as one of the dominant modes of transportation, ridesharing services must consider the interest of associated multiple stakeholders. Implementation of appropriate strategies to match drivers and passengers can provide balance in satisfying multiple stakeholders' concerns. In this section, existing literatures related to drivers and riders matching are reviewed.

There are two possible ways to match drivers and riders in the context of ridesharing: - (i) providing cruising routes to drivers in order to get passengers in those routes instead of serving specific pick-up requests, or (ii) assigning drivers to serve certain passenger requests with a specific route plan. Both approaches are focused on achieving different operational objectives (e.g., minimize VMT, maximize drivers' profit). Ge et al. (2010) developed an energy-efficient driver referral system by extracting traffic patterns from vehicle location traces to reduce energy consumption by optimizing travel distance, and suggested a sequence of passenger pick-up points for each driver. Hou et al. (2013) used a busy-link dominant strategy to address the taxi routing problem with an objective to minimize global vacancy rate (i.e., travelling distance with no onboard passenger to the total travelling miles) considering traffic conditions. Qu et al. (2014) developed a referral system to maximize taxi drivers' profits when following recommended routes by optimizing a net profit objective function. Historical taxi GPS traces were used to develop a 
road network as a graph representation and brute force strategy was used to create optimal driving routes. Due to the high computation time of graph-based approaches, a novel recursion strategy for quick and efficient optimal driving routes was proposed. Compared to other driver recommendation systems, this system could provide a complete driving route for drivers instead of recommending chain of discrete pick-up points only. Hwang, Hsueh and Chen (2015) developed a recommender system for the determination of the next-best profitable cruising locations for taxi drivers. A location-to-location graph known as the ON-OFF model was used to estimate expected fares for each trip. For this purpose, a driver experience factor was used in addition to three common factors (i.e., distance, waiting time and expected fare). The proposed driver recommendation systems discussed above were based on predicted demand, not actual demand, in an area. Moreover, mobilizing all drivers to the same areas could lead to a system imbalance, while passengers located in low-demand areas require more time for trips because of driver unavailability in the area.

The second type of driver recommendation systems is based on real-time exact requests, rather than allocating drivers to predicted high demand areas. Solving the ridesharing problem based on received requests is a kind of dial-a-ride problem. However, the ridesharing problem has additional constraints such as drivers' acceptable trip time windows, drivers' own origin and destination, which reduces the search space and complicates the problem. Armant and Brown (2014) used a mixed-integer programming formulation of the ridesharing problem applying two optimization techniques (i.e., linearization and symmetry breaking) to solve where drivers and riders could change their role (i.e., when ride demand is too high, some potential passengers can decide to act as drivers and provide rides, instead of requesting a ride). The limitation of this study was that the driving route between a pair of locations is similar regardless of the existing requests, and drivers do not have to cover any detour distance. Ehsani and Yu (2017) engineered the New York City Taxis and Limousine Commission dataset to estimate benefits of the proposed matching algorithm. Evaluation results predicted total system-wide VMT reduction with maximized participation in ridesharing though shared rides used by all users in dataset was unrealistic assumption. Biswas et al. (2017) focused on profit-maximization of a commercial rideshare service provider under a pricing model with detour-based discounts for passengers both for static (total detour) and dynamic (incremental detour) scenarios. New York City Taxi data was used to 
demonstrate their proposed greedy heuristic ride matching technique which resulted up to $90 \%$ optimal solutions in terms of profit compared to exponential-time optimal algorithm.

Several studies solved the ridesharing vehicle routing problem applying multi-objective optimization techniques. A routing optimization model of ridesharing taxi to minimize operational cost from the drivers' perspective and to maximize user satisfaction from a passengers' perspective is explored by Lin et al., 2012 and solved by applying a simulated annealing algorithm. It was found that ridesharing reduced taxi demand by $24 \%$ and system-wide VMT by $19 \%$. Multiobjective route planning algorithms were proposed by Herbawi and Weber, June 2011 and Herbawi and Weber, November 2011 for the dynamic multi-hop ridesharing problem where single rider's trip request was served by multiple drivers. Passengers' O-D trips for this ridesharing type is shared by multiple drivers who do not need to change their original route. But transferring passengers in between their trips may result inconvenience for them. In the improved version of the two previously mentioned algorithms, the authors considered multiple riders instead of a single rider sharing the trips with drivers along with time windows for pick-up and drop-off and drivers' detour requirements (Herbawi and Weber, 2012). A genetic algorithm was used to solve this ridesharing matching optimization problem considering multiple objectives i.e., minimizing travel time, minimizing travel distance, and maximizing the number of driver and rider matching. The proposed genetic algorithm was able to serve $73 \%$ of riders' request using trip demand scenario of Northeastern, Illinois when drivers with longer travel distances were selected from pool of travelers. But when drivers were chosen randomly, the algorithm was able to match $42 \%$ of riders' request satisfying all constraints with comparatively less sacrifice in terms of direct travel distance and direct travel time. Agatz et al. (2011) used an optimization approach tailored to the dynamic ridesharing problem and applied rolling horizon approach for solving by considering all the drivers and riders who offered or requested rides and previous unmatched rides by the time of execution. To evaluate the optimization model, a simulation study was conducted using 2008 Metropolitan Atlanta (GA, USA) travel demand data. Performance of ridesharing systems improved substantially in terms of the total number of matching and VMT savings comparing to a simple greedy matching rule. But according to the model formulation, a driver could only make one pickup and one delivery. This model does not allow travelling with additional riders in the vehicle, even the rider's pick-up location was in route of the driver. Jung et al. (2012) developed a dynamic shared taxi dispatch model based on two objectives- minimization of detour time and waiting time, 
and maximization of system-wide profits which included fixed revenue and distance-based revenue. Nearest Vehicle Dispatch (NVD), Insertion Heuristic (IS) and Hybrid Simulated Annealing (HSA) algorithms were used to solve the problem. Among them, HSA maximized the system's efficiency for real-time ridesharing in terms of drivers' profit, percentage of total request handled, ride time index and level of service index.

Wang et al. (2017) introduced the concept of stability in ridesharing by introducing a stability constraint in optimization of VMT savings, where the trips having negative VMT savings were not rejected, but rather, balanced with the trips that generated positive VMT savings. This approach can serve riders whose origins were away from the travel route and add additional VMT. One major concern of this approach is that some drivers would experience less satisfaction due to the additional travel distance.

Fixed meeting points could save en-route delays for in-vehicle passengers and drivers, increase the number of served passengers, and attain operational flexibility compared to door to door pick-up and drop-off in a ridesharing system. An extensive simulation study evaluated the meeting point concept based on real-world traffic data and found that meeting points increased the number of matches and decreased total VMT (Stiglic et al., 2015). Using this meeting point concept for ridesharing, Li et al., 2018 optimized total system cost which included travel time cost and cost of walking time to and from pick-up and drop-off points respectively. A tabu-based metaheuristic algorithm was used to solve the optimization problem. $2.7 \%$ to $3.8 \%$ travel time savings for a small-scale ridesharing system was reported. Kaan and Olinick, 2013 used park and ride locations as meeting points in developing the minimum cost vanpool assignment model. Assumption of common origins and destinations for all passengers was the limitation to this model's applicability for ridesharing.

While several past studies considered multiple objectives in developing ridesharing service models, no study evaluated the relative performance of multiple objectives considering the diverse interests of multiple stakeholders involved in transportation service related decision making. In this research, the meeting point concept is adopted in developing a ridesharing service considering four objectives and evaluating the relative performance of them. The findings of this research will help researchers and transportation decision makers develop systematically objective-oriented ridesharing matching system by considering system performance in terms of multiple stakeholders' perspectives. Multiple pick-up and drop-off options are considered in the ridesharing 
service model to maximize matching while optimizing certain objective function. Moreover, ridesharing services typically do not take drivers' origin and destination into consideration during ride matching; and drivers use their own judgement when accepting ride requests. This research considers drivers' origin and destination in developing ridesharing services. 


\section{CHAPTER 3: DRIVERS AND RIDERS MATCHING OPTIMIZATION MODELS}

In this research, four ridesharing drivers and riders matching objectives were selected to evaluate the relative performance of the system. Four selected objectives are: (i) minimization of systemwide passengers' waiting time, (ii) minimization of system-wide Vehicle Miles Travelled (VMT), (iii) minimization of system-wide detour distance, and (iv) maximization of system-wide drivers' profit. Each of the four objectives has potential to provide maximum benefit to few stakeholders and negatively effects other stakeholders.

\subsection{Selection of Objective Functions}

One of the significant benefits of ridesharing is the elimination of parking hassles in terms of parking cost and time to find parking spot in business districts. Also riders of ridesharing can perform other activities (e.g., reading papers/magazines) en-route instead of driving their own vehicles (Hensley, Padhi, and Salazar, 2017). However, ridesharing trips may require a longer trip time to pick-up and drop-off passengers, and longer waiting time for the ride to arrive at the trip origins. As a ridesharing service matching optimization objective (Objective \# 1), minimization of system-wide passengers' waiting time consists of waiting for driver at pick-up point, waiting time related to pick-up and drop-off of other passengers sharing the trip, and waiting time due to congestion, can ensure attractiveness of the service and travel convenience.

Relatively lower and stable fuel price, economic growth and rising household income are underpinning VMT increase over the last few decades (Piotrowski M., 2017). U.S. Energy Information Administration estimated 8,858 million miles of VMT per day by U.S motorists in 2018, which is 50\% more VMT per day compared to 1990 (US Energy Information Administration, n.d.). Higher VMT leads to congestion in existing roadways. One of the selling points of ridesharing service is the reduction in system-level VMT while serving equal numbers of passenger miles travelled. Thus, considering minimization of system-wide VMT (Objective \# 2) during matching riders and drivers can reduce the congestion level during rush hours, reduce emissions, fuel consumption and overall travel costs (Wang et al., 2017). From a transportation system operational perspective, transportation agencies prefer to reduce VMT by providing alternative transportation modes (e.g., ridesharing) without compromising economic activities of the region. 
Excessive detour distance in matching drivers and riders can affect drivers and on-board passengers' itinerary by increasing their total trip time. While detours are unavoidable in most of the ridesharing trips, it is important to consider both drivers' and passengers' maximum trip time flexibility to limit unexpected detour distance/time and ensure service quality and satisfaction. Drivers usually prefer passenger pick-up and drop-off points en-route between their origin and destination with minimum or no detour to pick-up and drop-off riders. On the other hand, on-board passengers also prefer drivers to travel the shortest route to their destination. Also, ridesharing service providers want a matching among drivers and riders with minimum detours as possible in order to improve users' satisfaction. Minimum detour can reduce travel time of a trip that not only increases satisfaction of users of ridesharing but also ensures more available drivers to serve new requests. Thus, minimization of detour distance (Objective \# 3) during matching is important to provide reliable service both to drivers and riders (Stiglic et al., 2015).

Attracting a pool of ridesharing drivers until the arrival of fully autonomous vehicles is critical for providing ridesharing services (Hensley, Padhi and Salazar, 2017). For ridesharing, a driver may need to perform a substantial amount of detour, and incur an additional trip time due to en-route passenger pick-ups and drop-offs. Ridesharing drivers may need to sacrifice more than riders in terms of travel convenience, status and freedom in return of travel cost savings/earning enabled by sharing trip cost with riders. Besides that, to attract drivers in order to participate in ridesharing an appropriate pricing mechanism that ensures sufficient drivers' profit is important. Thus, consideration of maximization of drivers' profit (Objective \# 4) as ridesharing matching objective can match riders with drivers in a way which will maximize drivers' profit.

\subsection{Model Parameters and Decision Variables}

Matching service providers for ridesharing will receive a stream of trip requests from participants and available drivers with their specific trip origin and destinations. Trip announcements can be classified in two parts- drivers' trip announcements and riders' trip requests. The matching agency will require diverse trip related information from each driver and rider to optimally develop matching trips. The trip related information of drivers and riders used in the development of ridesharing service considering four objectives are summarized in Table 1. 
Table 1 Information Required from Passengers and Drivers

\begin{tabular}{|c|c|}
\hline Information required from passengers & Information required from drivers \\
\hline $\begin{array}{l}\text { - } \text { Pick-up node/location } \\
\text { - } \text { Destination node/location } \\
\text { - No of passengers in a single request } \\
\text { - } \text { Time of request } \\
\text { - } \text { Maximum waiting time at pick-up } \\
\text { - } \text { point } \\
\text { Maximum acceptable ride time }\end{array}$ & $\begin{array}{l}\text { - } \text { Current location of vehicle } \\
\text { - Time of request } \\
\text { - No of seats available } \\
\text { - Drivers' destination node/location } \\
\text { - Acceptable latest arrival time at } \\
\text { destination }\end{array}$ \\
\hline
\end{tabular}

\subsubsection{Roadway network modeling parameters}

$L^{P}$ represents a link in the road network, where $\mathrm{P}$ varies from 1 to $m$, and $m$ is the total number of links in the study roadway network. Links are connected by nodes, $L_{n}^{P}$. Any request for ride from passengers is represented by $i$, where $\mathrm{R}$ is the set of all ride requests. Available drivers for rides are represented by $v_{t j}$, where $t$ is the type of vehicle that relates to corresponding vehicle passenger capacity. $V$ is the set of all available vehicles for ridesharing. Table 2 represents indices and parameters used in the formulation of ridesharing matching optimization problems.

\section{Table 2 Model Parameters}

\begin{tabular}{|l|c|l|c|}
\hline Parameters & Symbol & Parameters & Symbol \\
\hline Request ID & $i$ & Timestamp & $T$ \\
\hline Set of requests & $R$ & $\begin{array}{l}\text { Capacity of vehicle of type, } \mathrm{t} \text { and } \\
\text { vehicle \#, } \mathrm{j}\end{array}$ & $v_{t j}^{c}$ \\
\hline Origin of a request, $i$ & $o_{i}$ & Origin of a driver & $o_{v_{t j}}$ \\
\hline Destination of a request, $i$ & $d_{i}$ & Destination of a driver & $d_{v_{t j}}$ \\
\hline $\begin{array}{l}\text { Vehicle ID (type of vehicle, } t \\
\text { and vehicle ID, } j \text { ) }\end{array}$ & $v_{t j}$ & Number of passengers in request i & $N^{i}$ \\
\hline $\begin{array}{l}\text { Total number of vehicles } \\
\text { Length of a link, } L^{\mathrm{P}}\end{array}$ & $V$ & $\begin{array}{l}\text { Number of on-board passengers } \\
\text { in the vehicle before new pick-up }\end{array}$ & $N_{E}$ \\
\hline & $l_{L^{P}}$ & $\begin{array}{l}\text { Additional ride time due to an en- } \\
\text { route pick-up or drop-off at a node } \\
\text { by vehicle } v_{t j}\end{array}$ & $M_{L_{n}^{P}, v_{t j}}$ \\
\hline
\end{tabular}




\begin{tabular}{|c|c|c|c|}
\hline $\begin{array}{l}\text { Distance between two } \\
\text { consecutive nodes }\end{array}$ & $l_{L_{n,}^{P}, L_{n(\text { next })}^{P}}$ & $\begin{array}{l}\text { Distance between two nodes a and } \\
\mathrm{b}\end{array}$ & $T D_{a, b}$ \\
\hline Pick-up node of request $i$ & $L_{n, o_{i}}^{P}$ & $\begin{array}{l}\text { A node } L_{n}^{P} \text { on link } L^{P} \text { with } \\
\text { attribute, a }\end{array}$ & $L^{P}, L_{n}^{P}, a$ \\
\hline Destination node of request $i$ & $L_{n, d_{i}}^{P}$ & Congestion Delay & $C$ \\
\hline Fare per unit distance & $F$ & Fare inflation factor & $\alpha$ \\
\hline $\begin{array}{l}\text { Acceptable late pick-up time } \\
\text { of a request, } i\end{array}$ & $T_{l p_{i}}$ & $\begin{array}{l}\text { Acceptable late arrival time of } \\
\text { driver in vehicle, } v_{t j}\end{array}$ & $T_{l a_{v_{t j}}}$ \\
\hline $\begin{array}{l}\text { Number of on-board } \\
\text { passengers in a vehicle at any } \\
\text { node after pick -up and drop- } \\
\text { off }\end{array}$ & $N_{L_{n}^{P}, v_{t j}}$ & $\begin{array}{l}\text { Maximum travel time (allowed by } \\
\text { a passenger) }\end{array}$ & $T_{M}^{L_{n, d_{i}}^{P}, L_{n, o_{i}}^{P}}$ \\
\hline $\begin{array}{l}\text { Pick-up time of trip request } \mathrm{i} \\
\text { (in vehicle } v_{t j} \text { ) from origin } \\
\text { node } L_{n}^{P}\end{array}$ & $T_{i, v_{t j}, L_{n, o_{i}}^{P}}$ & $\begin{array}{l}\text { Drop-off time of trip request } \mathrm{i} \text { (in } \\
\text { vehicle } v_{t j} \text { ) at destination node } \\
L_{n}^{P}\end{array}$ & $T_{i, v_{t j}, L_{n, d_{i}}^{P}}$ \\
\hline $\begin{array}{l}\text { Starting time of vehicle } v_{t j} \\
\text { from its origin node } L_{n}^{P}\end{array}$ & $T_{v_{t j}, L_{n, o}^{P} v_{t j}}$ & $\begin{array}{l}\text { Arrival time of vehicle } v_{t j} \text { at its } \\
\text { destination node } L_{n}^{P}\end{array}$ & $T_{v_{t j}, L_{n, d_{v_{t j}}}}$ \\
\hline $\begin{array}{l}\text { Penalty for unassigned } \\
\text { passengers' waiting time }\end{array}$ & $U_{W}$ & $\begin{array}{lcc}\text { Penalty for } & \text { unassigned } \\
\text { passengers' VMT } & \end{array}$ & $D_{F}$ \\
\hline $\begin{array}{l}\text { Penalty for unassigned } \\
\text { passengers' detour distance }\end{array}$ & $D D_{F}$ & $\begin{array}{l}\text { Ridesharing fare in vehicle } v_{t j} \text { at } \\
\text { link between nodes } L_{n}^{P} \text { and } \\
L_{n(\text { next })}^{P}\end{array}$ & $R F_{L_{n}^{P}, v_{t j}}$ \\
\hline Fuel cost per mile & $\mathrm{f}$ & & \\
\hline
\end{tabular}

\subsubsection{Decision variables}

Following three decision variables are considered in this research: -

i. $y_{i, v_{t j}}$ provides decision on a match between a driver $v_{t j} \in \mathrm{V}$ with a request $\mathrm{i} \in \mathrm{R}$.

$\circ$ If the driver of vehicle $v_{t j}$ can serve the request $i, y_{i, v_{t j}}=1$

$\circ$ If the driver of vehicle $v_{t j}$ cannot serve the request $i, y_{i, v_{t j}}=0$.

ii. $X_{L_{n}^{P}, v_{t j}}$ represents if there is a pick-up or drop-off associated to a node for vehicle $v_{t j}$.

○ If there is a pick-up or drop-off request at a node for vehicle $v_{t j}, X_{L_{n}^{P}, v_{t j}}=1$.

$\circ \quad$ If there is no pick-up or drop-off request at a node for vehicle $v_{t j}, X_{L_{n}^{P}, v_{t j}}=0$.

iii. $\quad Z_{i}$ denotes if a request is served by a match. 
○ If there is no match available, $Z_{i}=1$.

○ If a request is assigned and $Z_{i}=0$.

\subsection{Model Formulation}

In this subsection, we formulate four objectives considered in this research to evaluate their relative efficiency in matching drivers and riders. The following assumptions were made in model formulation.

i. Each request will be fulfilled by one vehicle/driver.

ii. Unassigned requests will use an alternative mode of transportation.

iii. Request execution of drivers and riders will be static rather than dynamic. Available requests will be executed by available drivers at that time.

iv. For a particular request, delay due to other requests' pick-up and drop-off, will be counted at nodes other than pick-up and drop-off nodes of that particular request.

v. Vehicle will follow uniform speed. There will be no delay due to congestion.

vi. Fare will be shared by both drivers and passengers.

vii. Overall profit from a ridesharing trip will be considered as the reward for the driver.

viii. Pick-up and drop-off occur at nodes only.

ix. The problem formulations consider 'ride now' type of ride requests, and not applicable for "ride later" type of requests.

\subsubsection{Objective \# 1: Minimization of system-wide waiting time of passengers}

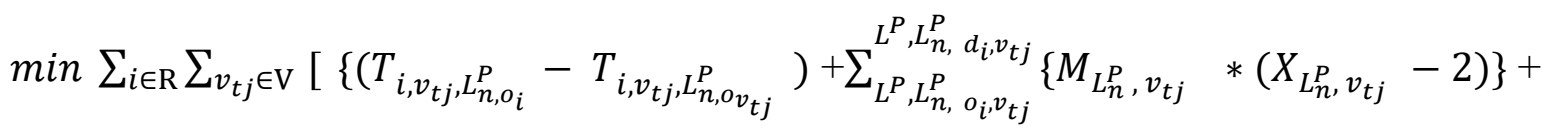

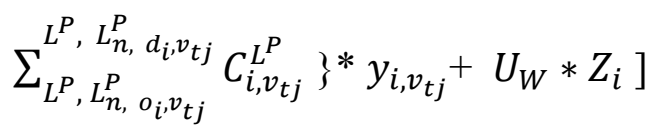

This objective function consists of four waiting time components. The first component represents the waiting time of ride request (consisting of single or multiple passengers) at the pickup node. The second component of the objective function represents waiting time related to the en-route pick-up and drop-off requests, which will be counted at nodes other than pick-up and drop-off nodes of the request concerned. The third component reflects the waiting time at congested locations. The fourth component represents waiting time penalty for unassigned 
passengers to account for the requests those are not matched with available drivers. Passengers of these unmet requests need to take alternate modes and that will increase passengers' system-wide waiting time and total travel time.

This objective function is subjected to following constraints

$T_{l p_{i}} \geq T_{i, v_{t j}, L_{n, o_{i}}^{P}}$ where $\forall v_{t j} \in \mathrm{V}, \forall \mathrm{i} \in \mathrm{R}$

$\sum y_{i, v_{t j}} * N^{i}+N_{E} \leq v_{t j}^{c} \quad$ where $\forall v_{t j} \in \mathrm{V}, \forall \mathrm{i} \in \mathrm{R}$

$\sum y_{i, v_{t j}}+Z_{i}=1$ where $\forall v_{t j} \in \mathrm{V}, \forall \mathrm{i} \in \mathrm{R}$

$T_{i, v_{t j}, L_{n, d_{i}}^{P}}-T_{i, v_{t j}, L_{n, o_{i}}^{P}} \leq T_{M}^{L_{n, d_{i}}^{P}, L_{n, o_{i}}^{P}}$ where $\forall v_{t j} \in \mathrm{V}, \forall \mathrm{i} \in \mathrm{R}$

$T_{v_{t j}, L_{n, d_{v_{t j}}}} \leq T_{l a_{v_{t j}}}$ where $\forall v_{t j} \in \mathrm{V}$

Constraint (1) guarantees that latest pick-up time acceptable to each request is not violated. Constraint (2) ensures that existing on-board passengers in a vehicle and new request assigned for the same vehicle never exceeds vehicle seating capacity. Constraint (3) ensures that each request is fulfilled by only one vehicle. Constraint (4) assures that the total ride time of any request does not exceed the maximum acceptable ride time of each passenger. Constraint (5) confirms that drivers' acceptable arrival time at his/her destination is satisfied.

\subsubsection{Objective \# 2: Minimization of system-wide vehicle miles traveled (VMT)}

$\operatorname{Min} \sum_{i \in \mathrm{R}} \sum_{v_{t j} \in \mathrm{V}}\left[\left\{y_{i, v_{t j}} *\left(\sum_{L^{P}, L_{n, o_{t}}^{P}{ }_{t j}}^{L^{P} L_{n}^{P}} l_{L_{n}^{P}, L_{n(\text { next })}^{P}}\right)\right\}+\left\{Z_{i} * D_{F} *\left(\sum_{L^{P}, L_{n, o_{i}}^{P}}^{L^{P}, L_{n}^{P}} l_{L_{n}^{P}, L_{n(n e x t)}^{P}}\right)\right\}\right]$

The first component of this objective function represents VMT by all assigned requests and second part represents the VMT by all unassigned requests. This objective function is also subjected to constraints (1) to (5) defined in first objective function.

\subsubsection{Objective \# 3: Minimization of system-wide detour distance}

$\min \sum_{i \in \mathrm{R}} \sum_{v_{t j} \in \mathrm{V}}\left[\left\{T D_{L^{P}, L_{n, o}^{P} v_{t j}, L^{P}, L_{n, d_{v_{t j}}}^{P}(\text { after })}-T D_{L^{P}, L_{n, o_{v} j}^{P}, L^{P}, L_{\left.n, d_{v_{t j}} \text { (before }\right)}}\right\} * y_{i, v_{t j}}+\right.$ $\left.\left(T D_{L^{P}, L_{n, o_{i}}^{P}, L^{P}, L_{n, d_{i}}^{P}} * Z_{i} * D D_{F}\right)\right]$ 
This objective function is the sum of total detour distance travelled by all drivers and unassigned requests. This objective function is also subjected to constraints (1) to (5) defined in first objective function.

\subsubsection{Objective \# 4: Maximization of system-wide drivers' profit}

$\max \sum_{i \in \mathrm{R}} \sum_{v_{t j} \in \mathrm{V}}\left[\sum_{L^{P}, L_{n, o_{v}}^{P} L_{t j}^{P}}^{L_{n, d_{t j}}^{P}}\left\{R F_{L_{n}^{P}, v_{t j}} * l_{L_{n,}^{P} L_{n(n e x t)}^{P}} * N_{L_{n}^{P}, v_{t j}} *(1+\alpha)\right\}\right]$

where $R F_{L_{n}^{P}, v_{t j}}=\frac{l_{L_{n}^{P} L_{n(n e x t)}^{P} *(F-f)}}{N_{L_{n}^{P}, v_{t j}}+1}$

Total base fare of a trip between two nodes is evenly distributed among on-board passengers and driver. In commercial ridesharing the total fare is distributed only among passengers. But in this study, we have assumed that drivers will also carry an equal portion of the fare with the concept that drivers share the seat of their vehicles with other riders between their own trip's origin and destination. This objective function is also subjected to constraints (1) to (5) defined in first objective function with one additional constraint for fare inflation factor (Ma et al., 2013).

$\alpha \geq 0$ where $\alpha \in(0,1)$ is the fare inflation factor

Fare inflation factor increases the total trip fare by certain percentage to ensure drivers monetary benefit for providing ridesharing service. These additional fares (beyond true trip cost) collected from shared riders act as an incentive for drivers to participate in ridesharing service. Fare inflation factor should be adjusted in such a way that there will be sufficient volume of drivers and riders in the system to provide a reliable service and ensure monetary benefit to drivers and cost savings to riders. An inappropriate fare inflation factor may result in more ridesharing drivers or more ridesharing riders compared to a balanced number of drivers and riders that can satisfy all trips. In this chapter formulation of four objective functions considered in matching riders and drivers are defined and their performance evaluation is presented in next chapter. 


\section{CHAPTER 4: ANALYSIS AND RESULTS}

To evaluate the performance of four objectives in providing ridesharing service, a hypothetical grid roadway network was used in this research (Figure 1). The size of the grid network is 10 miles $\times 10$ miles with spacing of one mile between parallel links (i.e., roadways). It was assumed that all 121 nodes where cross streets intersect can be used for passenger pick-ups and drop-offs (i.e., meeting points). The length of each link between two consecutive nodes is one mile. To develop a hypothetical ridesharing system, it was assumed that there were 16 ride requests consisting of 24 passengers ( 8 requests with a single rider and 8 requests with two riders) where six drivers were available to serve those requests. The pick-up and drop-off locations for ride requests and drivers' origins and destinations were chosen randomly before developing rider and driver matching considering each optimization objective. Figure 1 illustrates the hypothetical grid roadway network along with each driver's trip origin and destination (O-D) and each passenger request's pick-up and drop-off location at the beginning of ride matching. Table 3 summarizes the ride sharing system parameters assumed during matching objectives' performance evaluation.

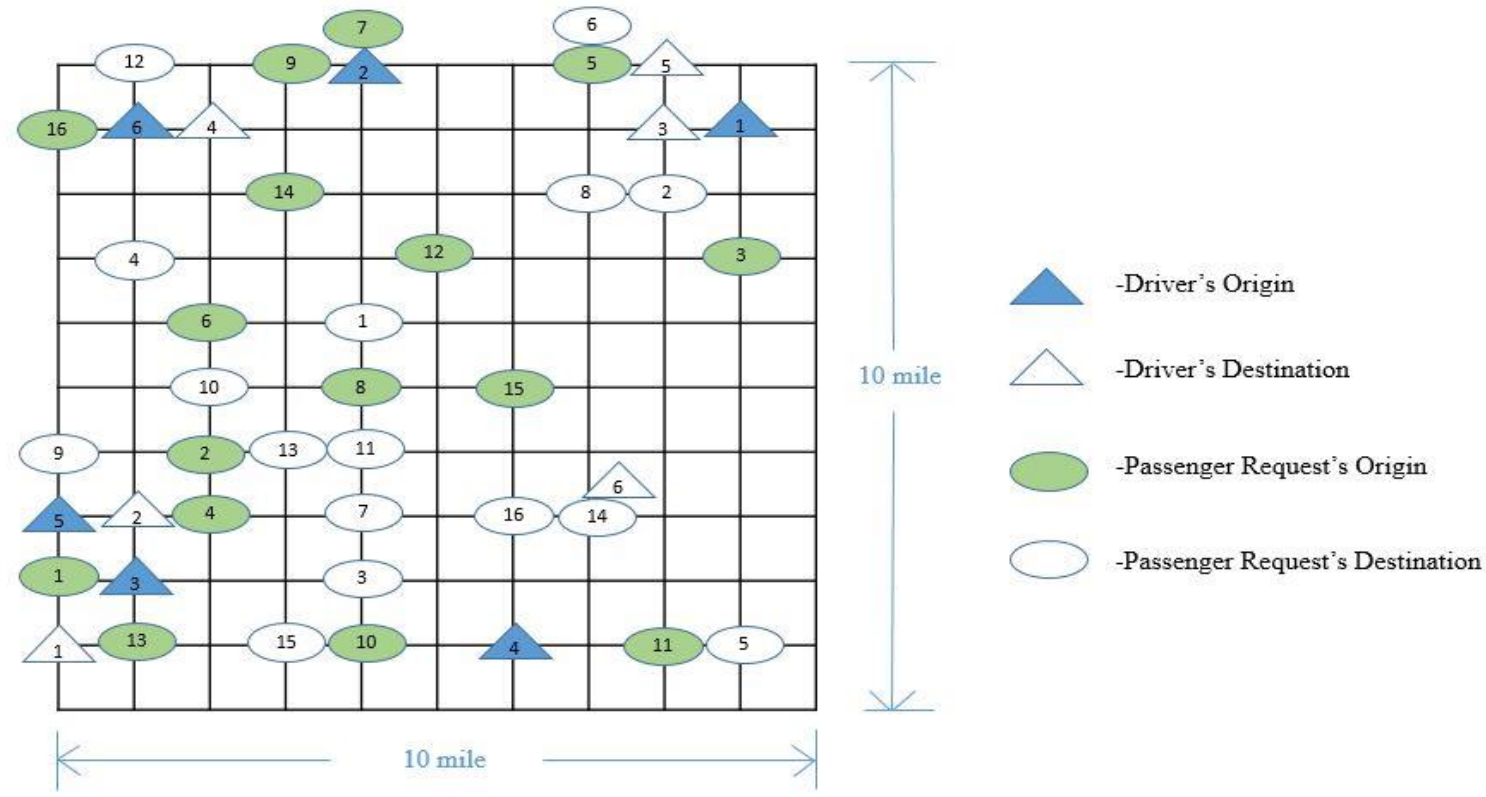

Figure 1 Hypothetical Grid Network, Drivers' O-D and Passenger Requests' Pick-up and Dropoff Locations Used in Drivers' and Passengers' Matching Optimization 
Table 3 Ridesharing System Parameters Assumed during Matching Objectives' Performance Evaluation

\begin{tabular}{|l|c|}
\hline \multicolumn{1}{|c|}{ Ridesharing System Parameters } & Value \\
\hline Network Size & 10 mile $\times 10$ mile \\
\hline Link length, $l_{L^{P}}$ & $30 \mathrm{sec}$ \\
\hline Pick-up and drop-off time at a node, $\mathrm{M}$ & 6 \\
\hline Number of drivers, V & 16 \\
\hline Number of requests, $\mathrm{R}$ & 24 \\
\hline Number of Passengers & $70 \%$ \\
\hline $\begin{array}{l}\text { Percentage of maximum extra ride time } \\
\text { allowed between O-D (passengers) }\end{array}$ & $100 \%$ \\
\hline $\begin{array}{l}\text { Percentage of maximum extra ride time } \\
\text { allowed between O-D (drivers) }\end{array}$ & 15 minutes \\
\hline Maximum waiting time at pick-up point & 0 minute \\
\hline Congestion delay on any link, C & $\$ 2$ \\
\hline Fare per mile, F & 0.4 \\
\hline Fare Inflation factor, $\alpha$ & 10 cents \\
\hline Fuel cost per mile, f & 40 mph \\
\hline Average speed of vehicles & 4 \\
\hline Passenger capacity of a vehicle except driver, & \\
$v_{t j}^{c}$ & \\
\hline
\end{tabular}

To accommodate ridesharing, both drivers and riders need to allow extra time for detours required to pick-up and drop-off passengers. Drivers require a longer trip time compared to passengers as they need to cover all detours, pick-ups, and drop-offs during the trip and reach their destination after serving all assigned trips. For this analysis, we assumed that passengers and riders will allow a maximum of $70 \%$ and $100 \%$ extra ride time respectively. Further, it was assumed that there was no congestion on the roadways that could increase trip time. Maximum waiting time acceptable by passengers at pick-up points was assumed to be 15 minutes. A 2016 survey in Ohio reported that more than $50 \%$ of the public transit riders routinely waited more than 30 minutes 
(Litman T., 2017). To enable ridesharing between available drivers and passenger requests, a set of possible routes between origin and destination of each driver was generated. Then four objective functions were optimized separately to select the best combination of routes for all drivers from the set of generated routes that provide the best performance for each objective function. In this process four routing combinations of six drivers from four objective functions' optimization were obtained separately. This study however has limitation in terms of generating optimal combination of routes for each objective optimization. The obtained vehicle routing combination from each objective function's optimization was best for the set of generated routes. Subsection 4.1 presents the performance of ridesharing services when the system was optimized for only one objective at a time.

\subsection{Impact of Ridesharing Matching Objectives on Travel Parameters}

Ridesharing service was optimized to match riders and drivers considering four objectives defined earlier in section 3.3. In this section, the performance of each objective in terms of system-wide passengers' waiting time, VMT, detour distance, and drivers' profit are presented and compared. In addition, matching percentage (percentage of total passengers served), passengers' average travel cost savings, and two performance indices (waiting time index and ride time index) were calculated and are discussed for all objectives.

Figure 2 summarizes the performance of Objective 1 (minimization of system-wide waiting time) when ridesharing serviced was optimized for Objective 1 (bar marked red), and other three objective functions. As expected, Objective 1 performance degraded when ridesharing optimization was done considering other three objective functions. The best value for the objective 1 was 118 minutes for the example ridesharing scenario and set of generated routes. The performance of the objective function degraded by $11 \%$ when the system was optimized for Objective 2 (minimization of system-wide VMT). Similarly, performance of the Objective 1 degraded by $20 \%$ and $23 \%$ when the system was optimized for Objectives 3 and 4, respectively.

Figure 3 summarizes the performance of Objective 2 (minimization of system-wide VMT) when the system was optimized for all four objectives. The best performance of Objective 2 was 131 miles for the set of generated routes when the system was optimized for Objective 2. The performance of Objective 2 degraded by 3\%, 4\% and $8 \%$ when the system was optimized for Objective 1, 3 and 4 respectively. 
Figure 4 represents the performance of Objective 3 (minimization of system-wide detour distance) when the system was optimized for all four objectives. The best value of Objective 3 was 19.8 miles of detour for the set of generated routes when the system was optimized for Objective 3. The value of Objective 3 was decreased by $40 \%, 21 \%$ and $76 \%$ when the system was optimized for Objective 1, 2 and 4, respectively.

From Figure 5 it is observed that the best value of the drivers' profit (Objective 4) was $\$ 140.98$ for the set of generated routes when the system was optimized for Objective 4. Performance of Objective 4 was degraded from $7 \%$ to $21 \%$ when the system was optimized for other objectives.

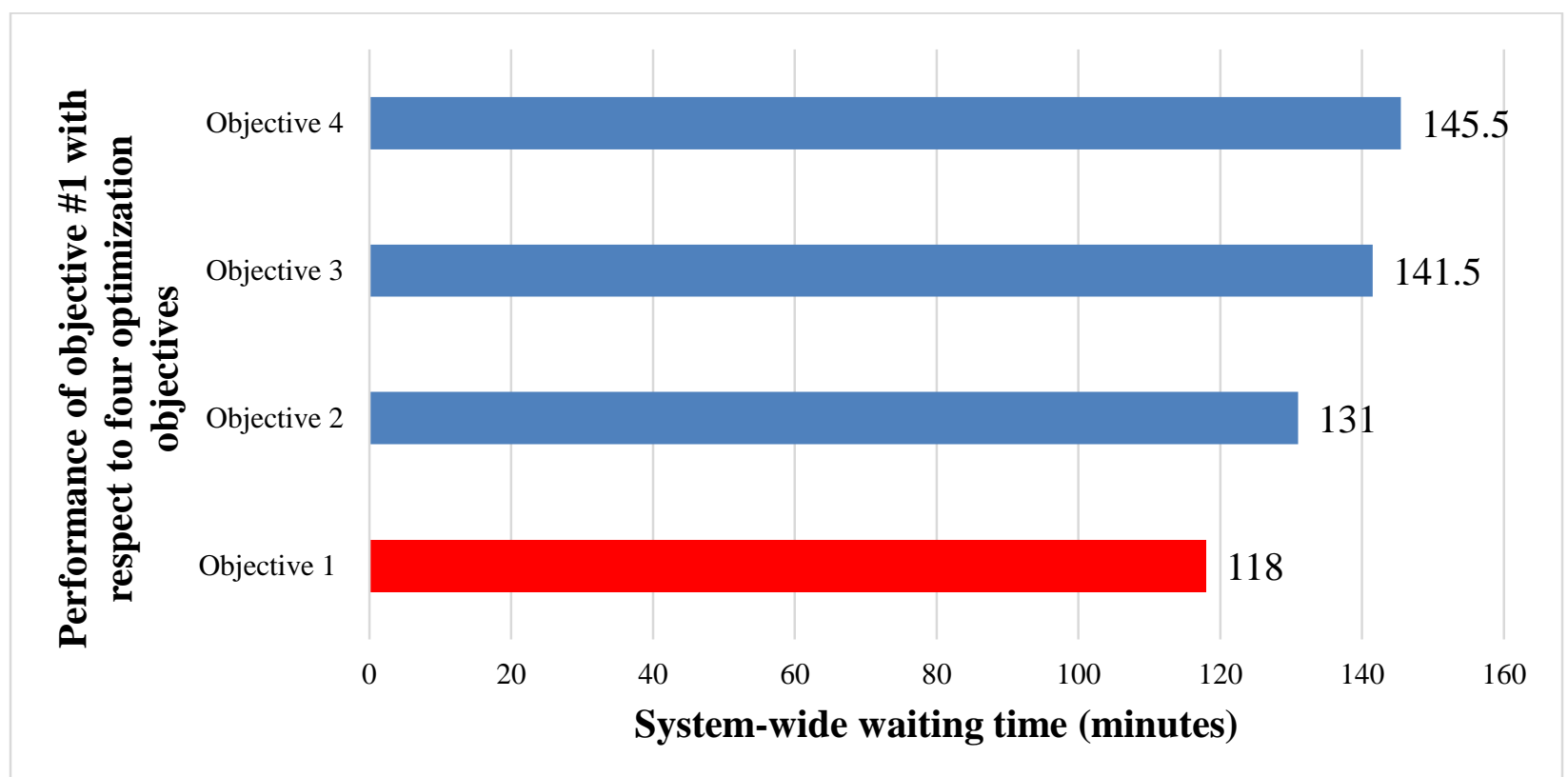

Figure 2 Comparison of Four Optimization Objectives' Performance in terms of System-wide Passengers' Waiting Time 


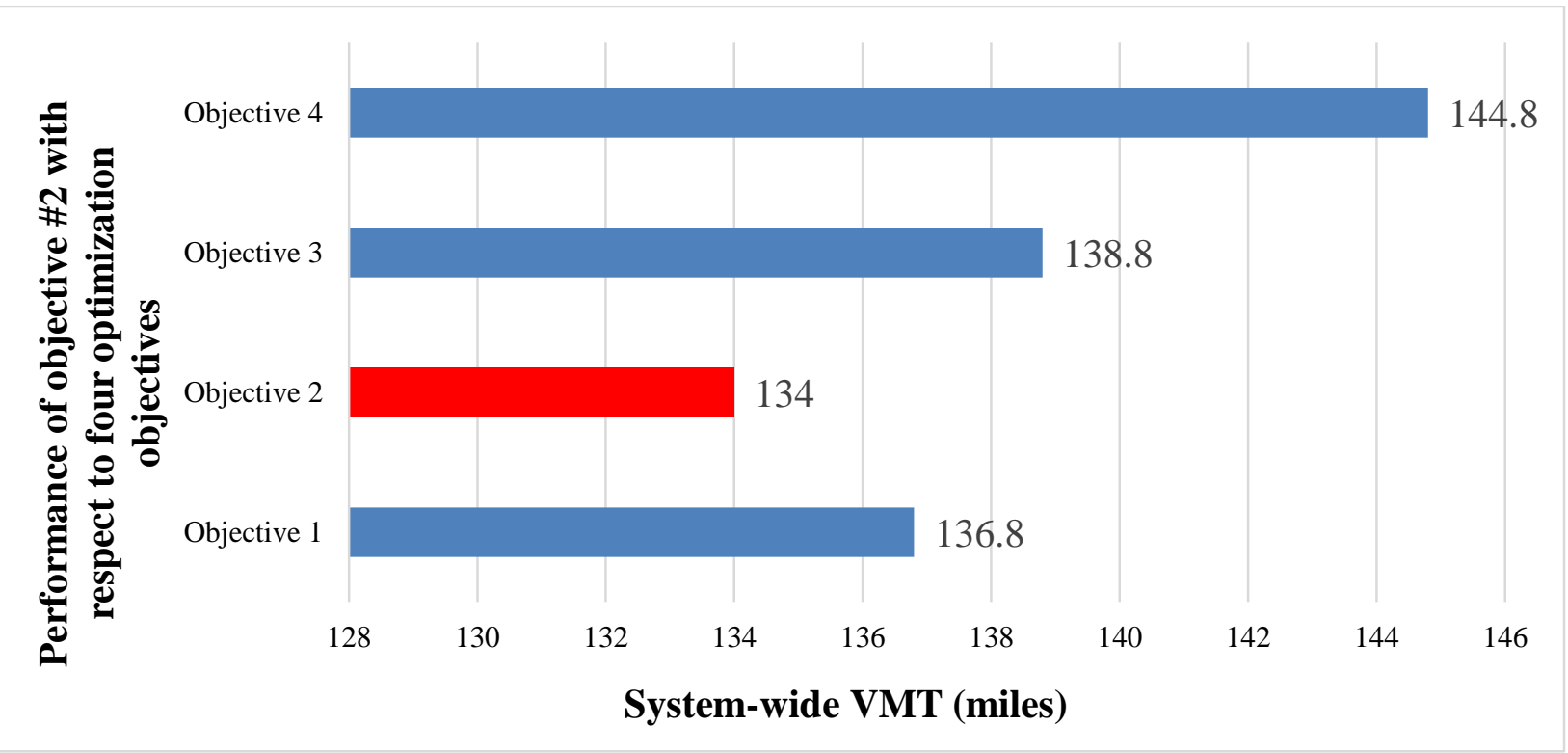

Figure 3 Comparison of Four Optimization Objectives' Performance in terms of System-wide VMT

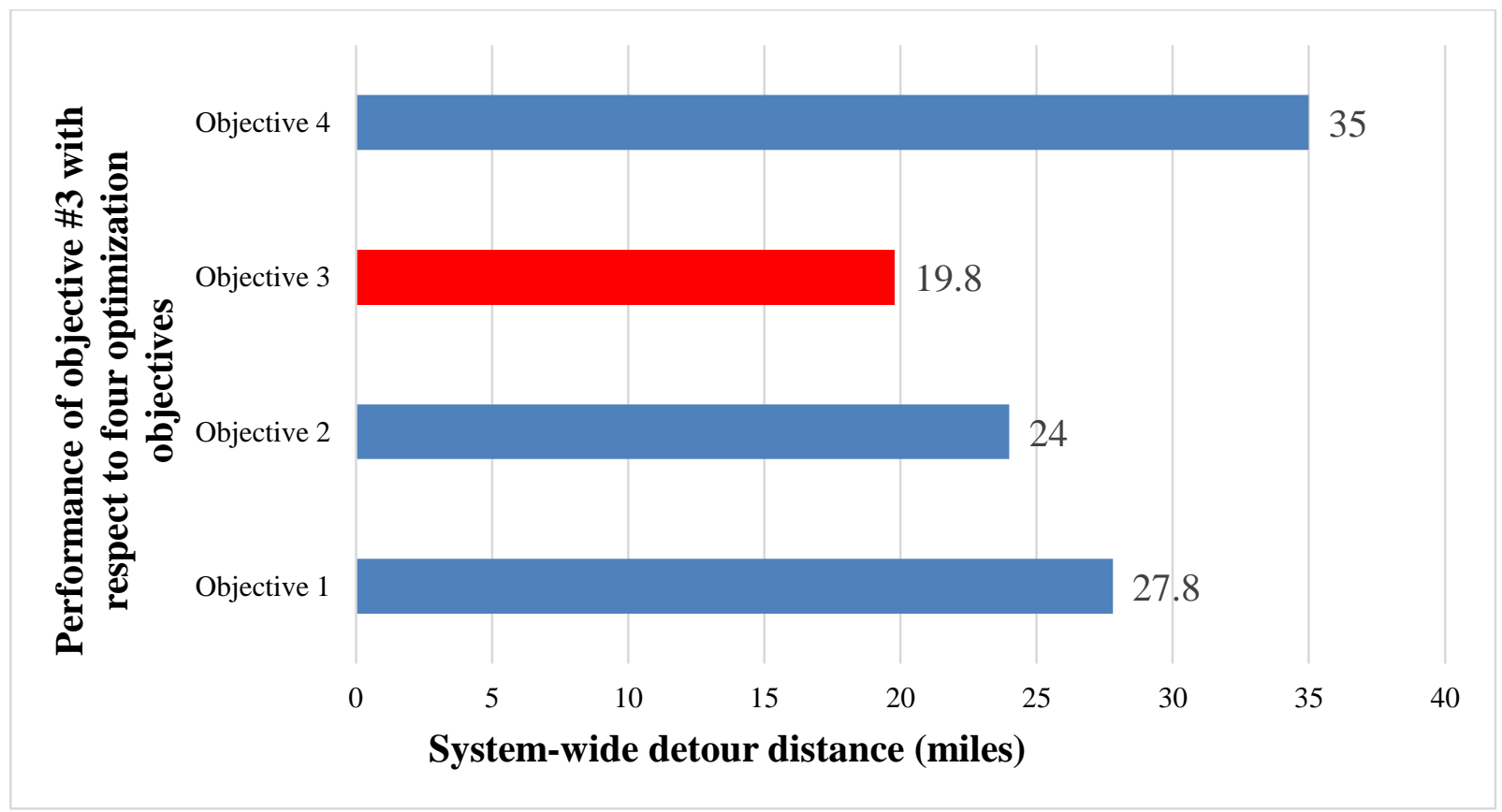

Figure 4 Comparison of Four Optimization Objectives' Performance in terms of System-wide Detour Distance 


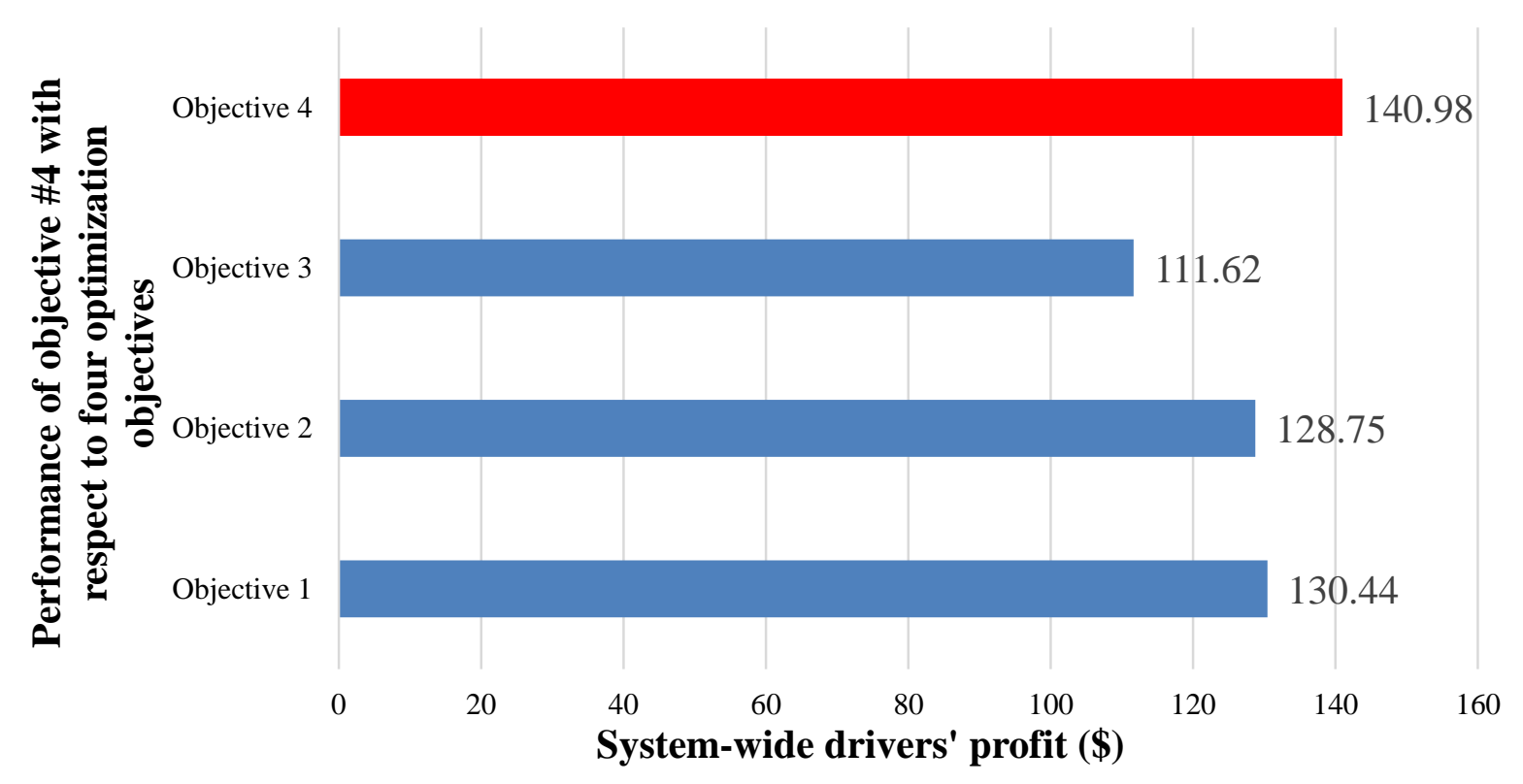

Figure 5 Comparison of Four Optimization Objectives' Performance in terms of System-wide Drivers' Profit

\subsubsection{Percentage of passengers served}

Figure 6 shows the percentage of trips served when the system was optimized for four different objectives separately. In terms of passenger handling capacity of four objectives, minimization of system-wide waiting time of passengers (Objective \# 1) and minimization of system-wide VMT (Objective \# 2) both served $75 \%$ of total requests. System-wide detour distance minimization (Objective \# 3) served the least percentage of passengers ( 67\%) (Figure 6) as this objective allowed limited flexibility in terms of detours. Drivers will be directed to take the route with minimum deviation from the shortest path between their origin and destination under this objective. Moreover, no objective could serve all the passenger requests satisfying optimization model constraints associated with each objective function. Three potential strategies can be implemented to serve more passenger requests:

(i) Rather than achieving optimal solution, near optimal solution can be used to serve more passengers by accepting a certain amount of sacrifice from optimal performance of each objective (Wang et al., 2017). Though, this strategy may not generate optimal performance, it can lead to mobility equity for passengers without personal vehicles who rely on ridesharing.

(ii) Switching role concept can be used to maintain balance in riders and available drivers to serve requests. In this concept, some users of ridesharing system switch their role between 
drivers and passengers (Armant and Brown, 2014). For example, some passengers can take the role of drivers to increase availability of drivers in order to serve excess ride requests.

(iii) Matching agency can adjust fare among different locations in order to divert drivers to high demand locations. Price should be adjusted in a way that does not discourage passengers. In today's ridesharing context, this concept is known as surge pricing. When there is high demand from passengers in a particular area, ridesharing companies, in order to serve more passengers, adjust prices to encourage more drivers to get to that area (Harolds S., 2018; Dustin, 2017).

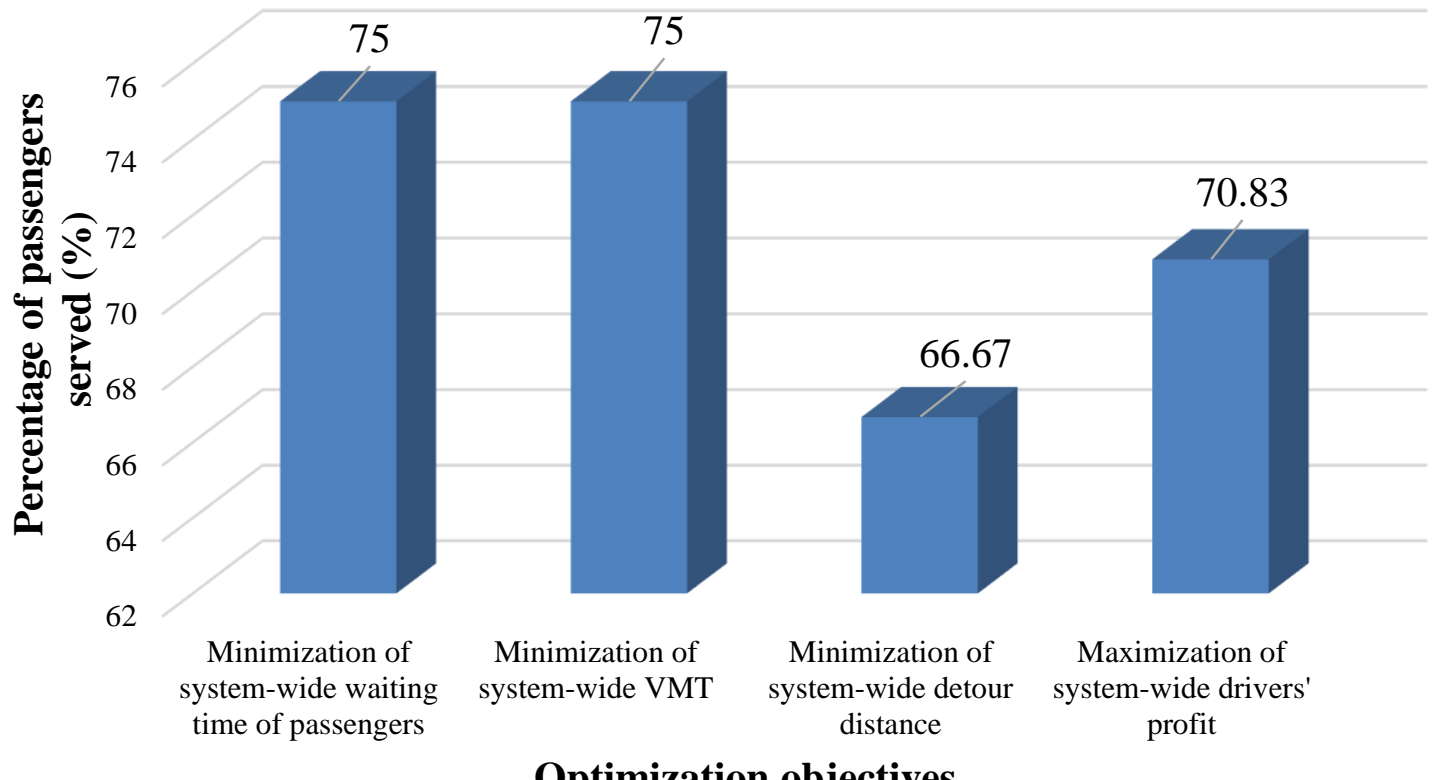

Optimization objectives

Figure 6 Percentage of Passengers Served through Optimization of Different Objectives

\subsubsection{Passengers' average travel cost savings}

Travel cost savings in ridesharing environment primarily depends on volume of ridesharing passengers, fare per mile, fuel cost per mile and fare inflation factor. Technology adoption (i.e. electric vehicles, connected and automated vehicle technology) have the potential to significantly decrease cost of vehicle operation and maintenance (Bagloee et al., 2016; Piotrowski M., 2017) in future ridesharing service. Travel cost savings of passengers and drivers is one of the main incentives of ridesharing. Performance evaluation of the four objectives considered in this research revealed that the maximization of system-wide drivers' profit (Objective 4) provided maximum 
cost savings to passengers (Figure 7). In this case, ridesharing system optimized for Objective 4 generated $\$ 10.34$ per passenger savings compared to a direct taxi service between passengers' origin and destination. To gain maximum profit, drivers needs to share rides with the maximum number of passengers (not exceeding vehicle capacity), and passengers can save travel cost as more passengers share total trip costs, which make per person travel cost lower. System-wide passengers' waiting time minimization (Objective 1) provided the least savings ( $\$ 8.73$ per passenger). Optimization of the ridesharing service for Objective \#1 prevented drivers from providing rides to those passengers who required significant amount of pick-up time due to long detours, which led to less number of passengers in each vehicle to share total trip cost.

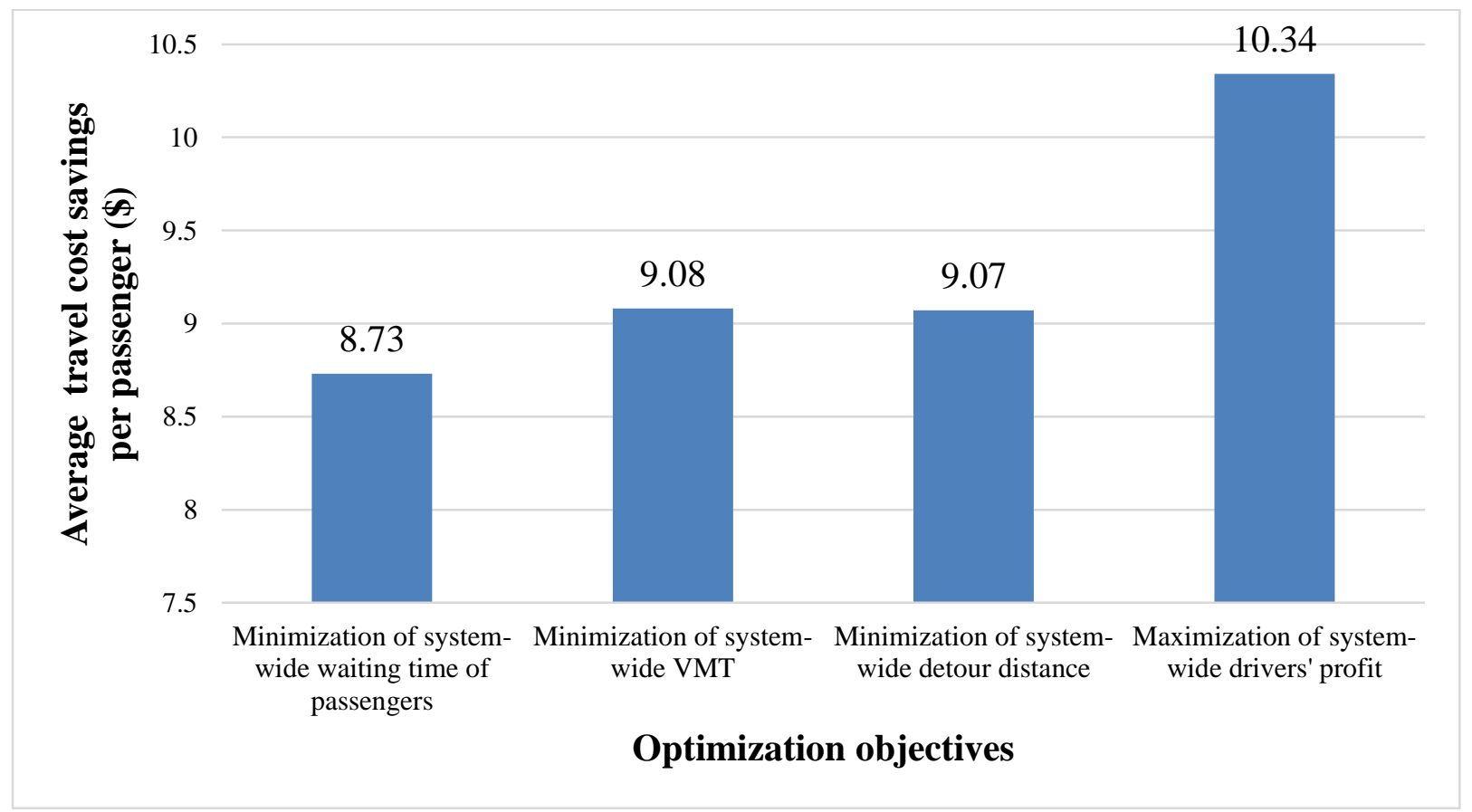

Figure 7 Average Travel Cost Saved Per Passenger for Four Objectives

\subsubsection{Performance of objectives in terms of ride time index and waiting time index}

Two trip efficiency related indices (i.e., waiting time index and ride time index) were calculated to measure the ridesharing system efficiency for four objectives. Waiting time index, referred as level of service index in Jung et al., 2013 is the ratio of average passenger waiting time at pick-up locations in ridesharing services and average ride time between origin and destination without any delay. The ride time index is the ratio of average passenger ride time in ridesharing service and the average ride time of passengers between origin and destination without any delay. Here, delay 
indicated the extra riding time (e.g., delay due to pick-up and drop-off, congestion and detour) compared to ideal travel conditions without any delay.

Waiting Time Index $=\frac{\text { Average passenger waiting time at pick-up location for ridesharing }}{\text { Avearage ride time between O-D in ideal conditions without any delay }}$

Ride Time Index $=\frac{\text { Average passenger ride time between } \mathrm{O}-\mathrm{D} \text { during ridesharing }}{\text { Avearage ride time between } \mathrm{O}-\mathrm{D} \text { in ideal conditions without any delay }}$

Minimization of system-wide waiting time (Objective \# 1) performs best in terms of waiting time index (Figure 8). For the ridesharing service system scenario considered in this study, passengers waiting at pick-up locations was equivalent to $39 \%$ of direct travel time between trip's origin and destination, and could discourage users to participate in ridesharing. This higher waiting time was primarily because of relatively short travelling distances between O-D pairs considered in the study ridesharing scenario. For same amount of waiting time for longer travelling distances, waiting time index will be lower and will be less discouraging to users.

System- wide detour distance minimization objective performs best in terms of ride time index. Passengers incur approximately $16 \%$ more travel time for participating in ridesharing services, which is the lowest compared to service optimization considering the other three objectives (Figure 9). Minimization of system-wide detour distance and minimization of systemwide VMT perform well among the four objectives in terms of ride time index (1.16 and 1.18 respectively), and system-wide waiting time minimization of passengers and system-wide drivers' profit maximization perform relatively worse (1.24 and 1.23 respectively). 


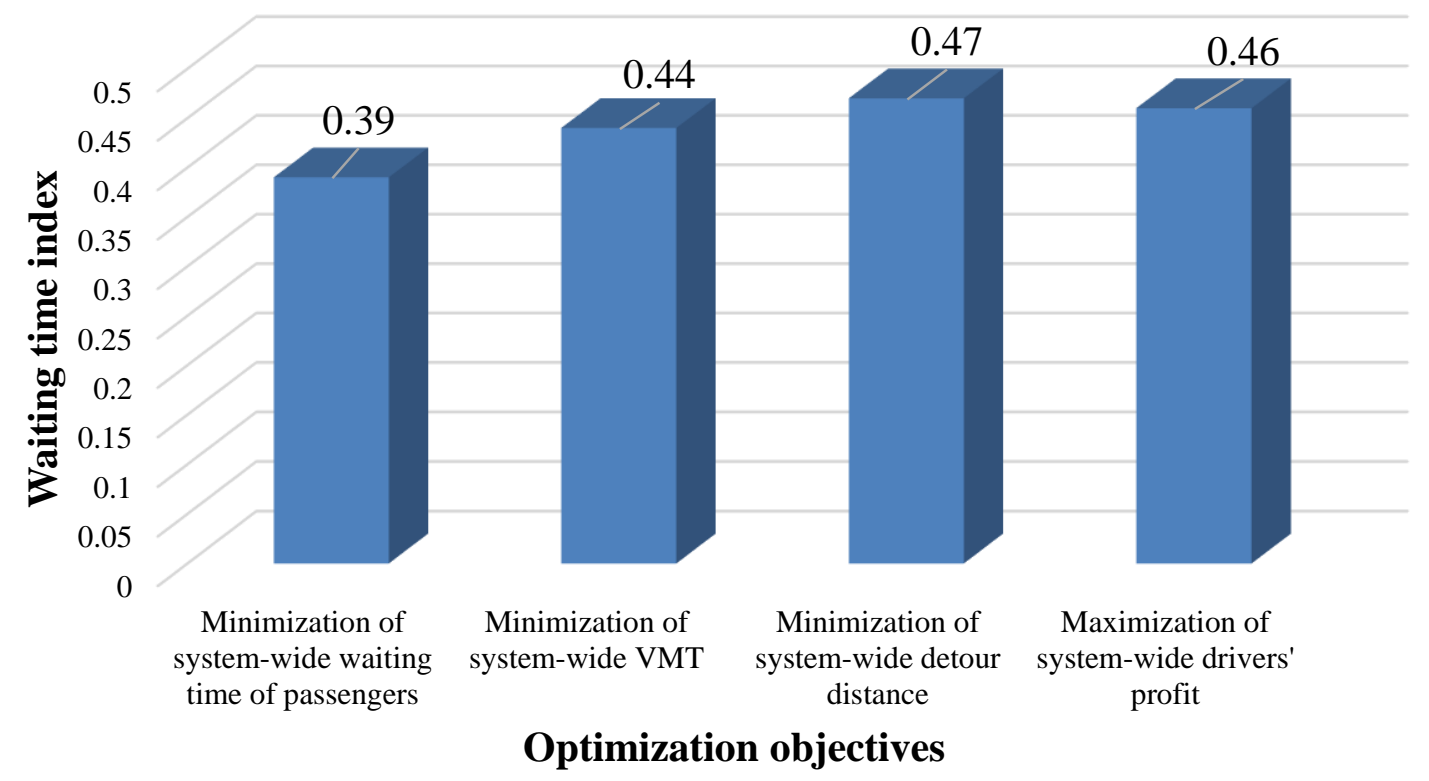

Figure 8 Waiting Time Index of Different Optimization Objectives

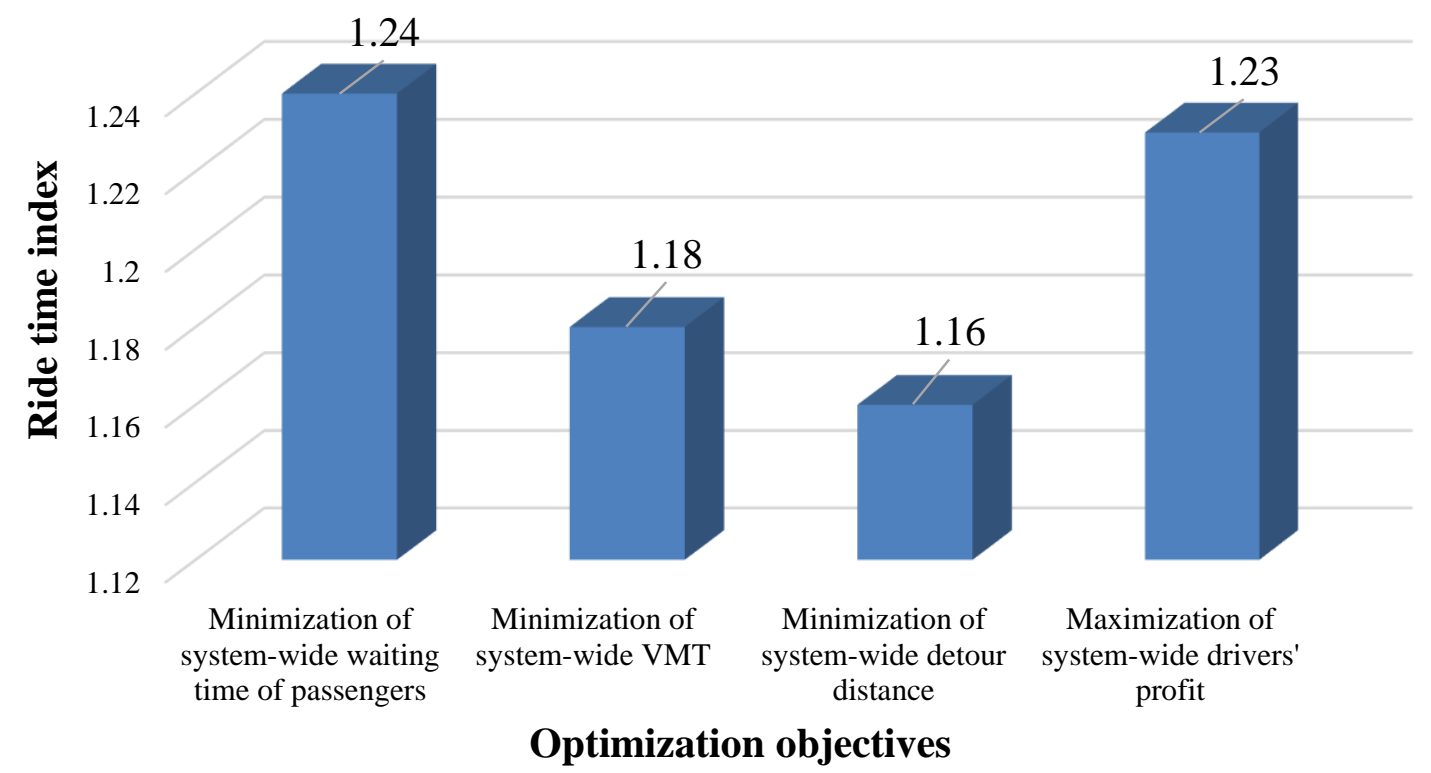

Figure 9 Ride Time Index of Different Optimization Objectives 


\subsection{Tradeoff between Four Objectives}

Development of any mobility service has consequences that impacts many stakeholders within the service jurisdiction. For example, while ride hailing services provide a new form of mobility through a mobile app, it was reported that ride hailing services contribute to congestion in major cities (LeBlanc, 2018). Thus, before implementation of any mobility services, it is important to assess the relative tradeoffs between the multiple objectives related to diverse stakeholders in order to ensure that some stakeholders are not adversely affected. Here, we compare the ridesharing system performance when the system was optimized for one matching objective by considering the tradeoffs that needed to be accepted in terms of the other three objectives (Figure 10 to Figure 13).

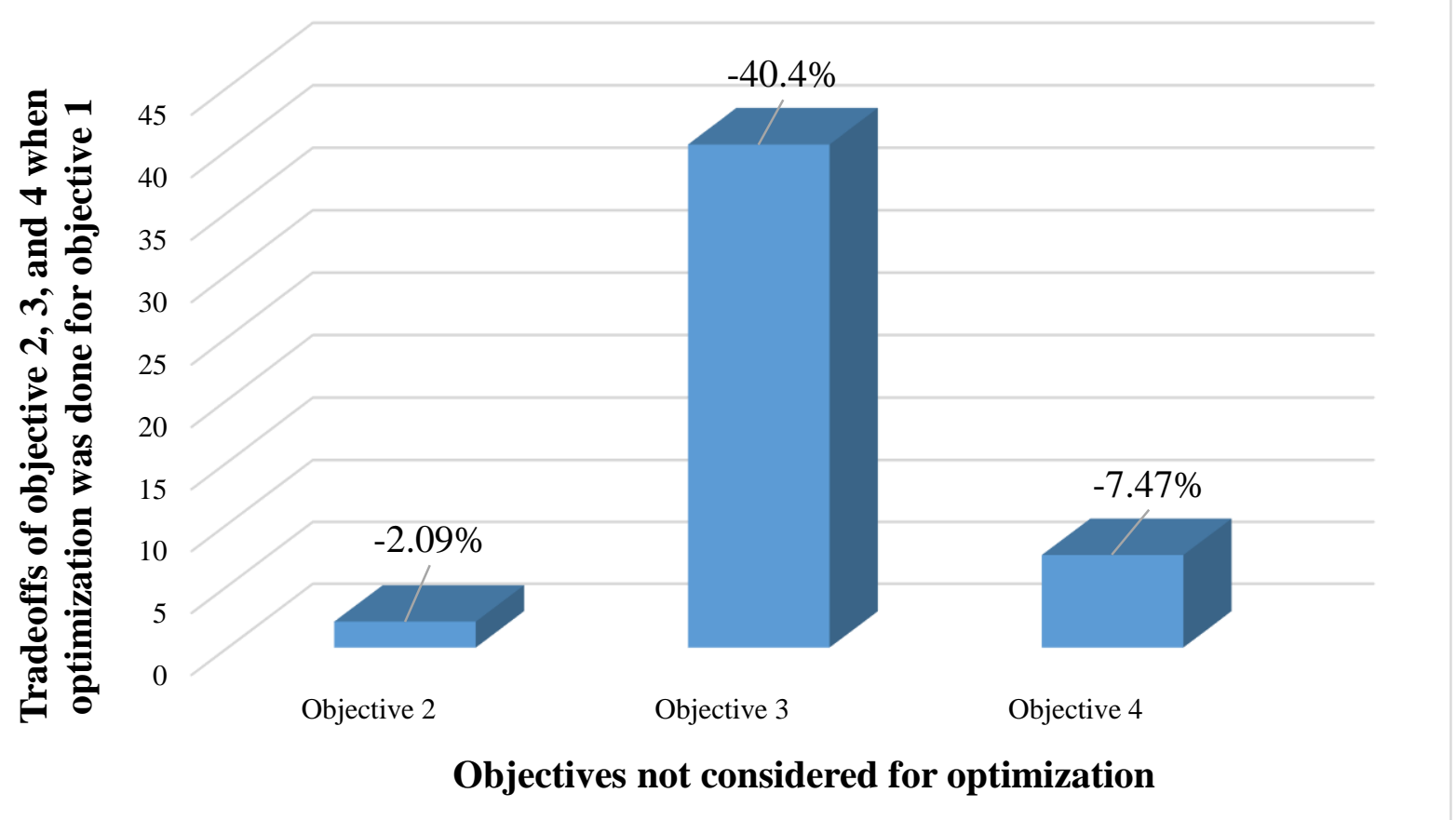

Figure 10 Tradeoff in terms of Three Objectives When Drivers' and Passengers' Matching Was Optimized for System-wide Passengers' Waiting Time 


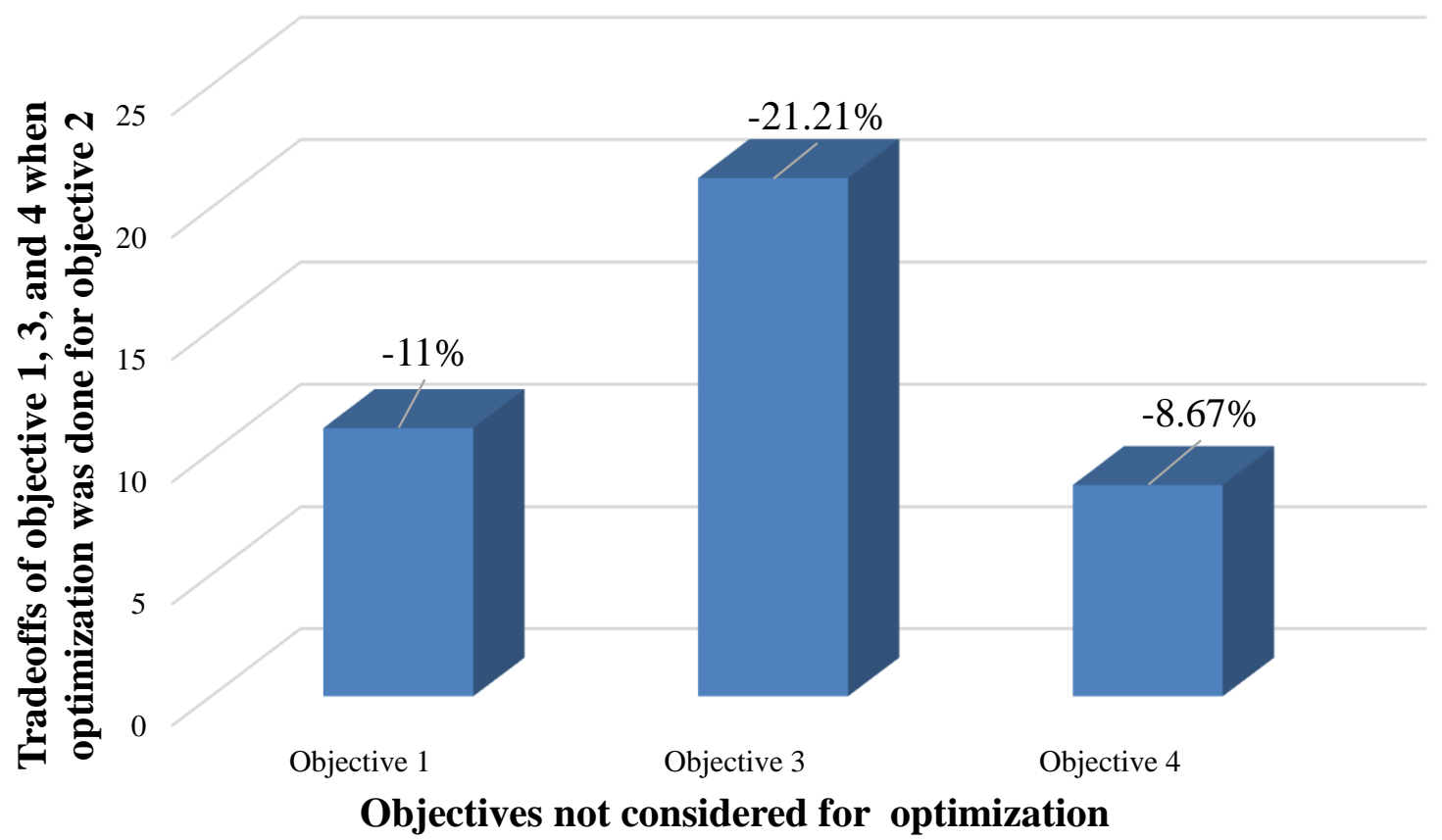

Figure 11 Tradeoff in terms of Three Objectives When Drivers' and Passengers' Matching Was Optimized for System-wide VMT

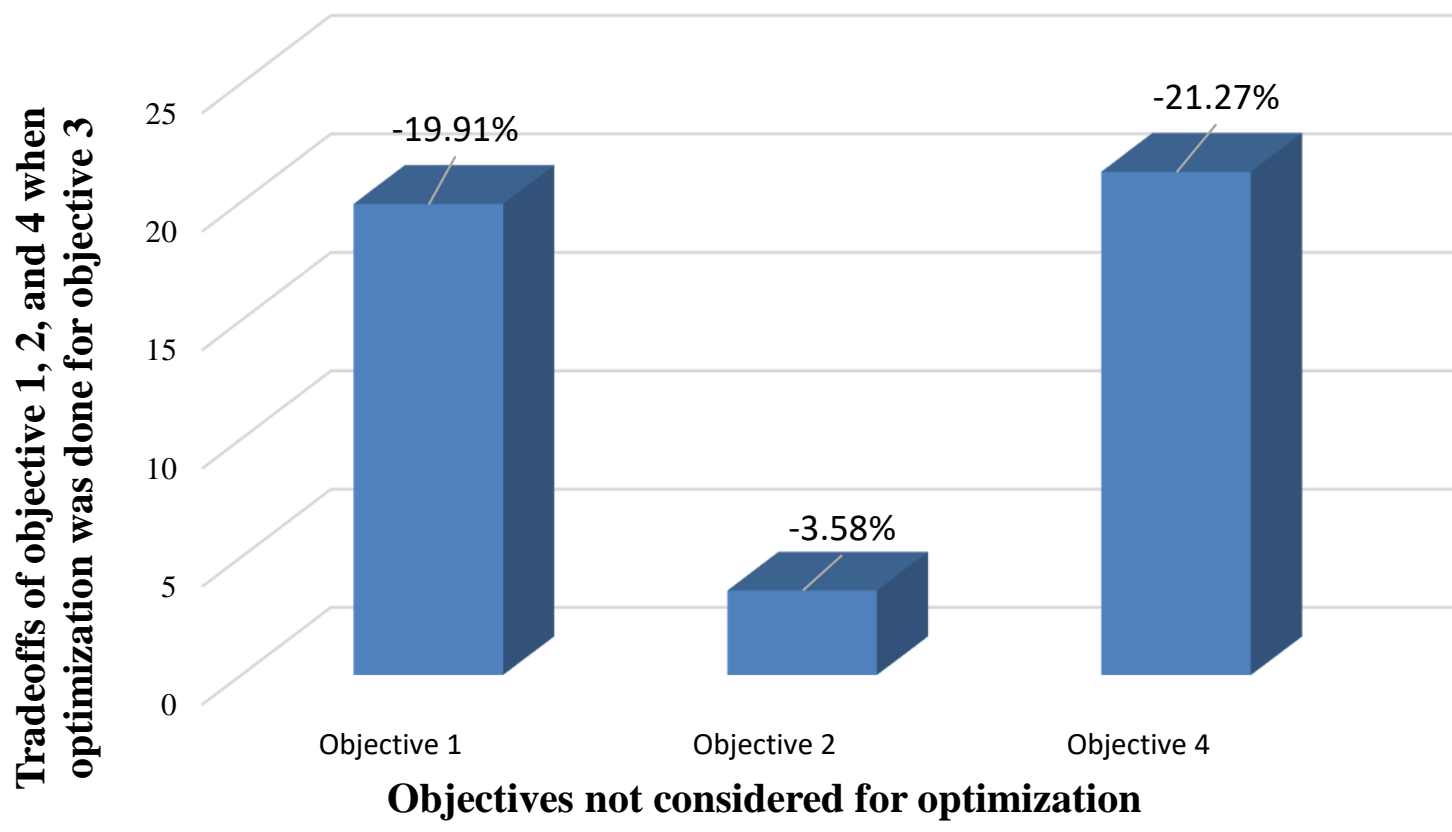

Figure 12 Tradeoff in terms of Three Objectives When Drivers' and Passengers' Matching Was Optimized for System-wide Detour Distance 


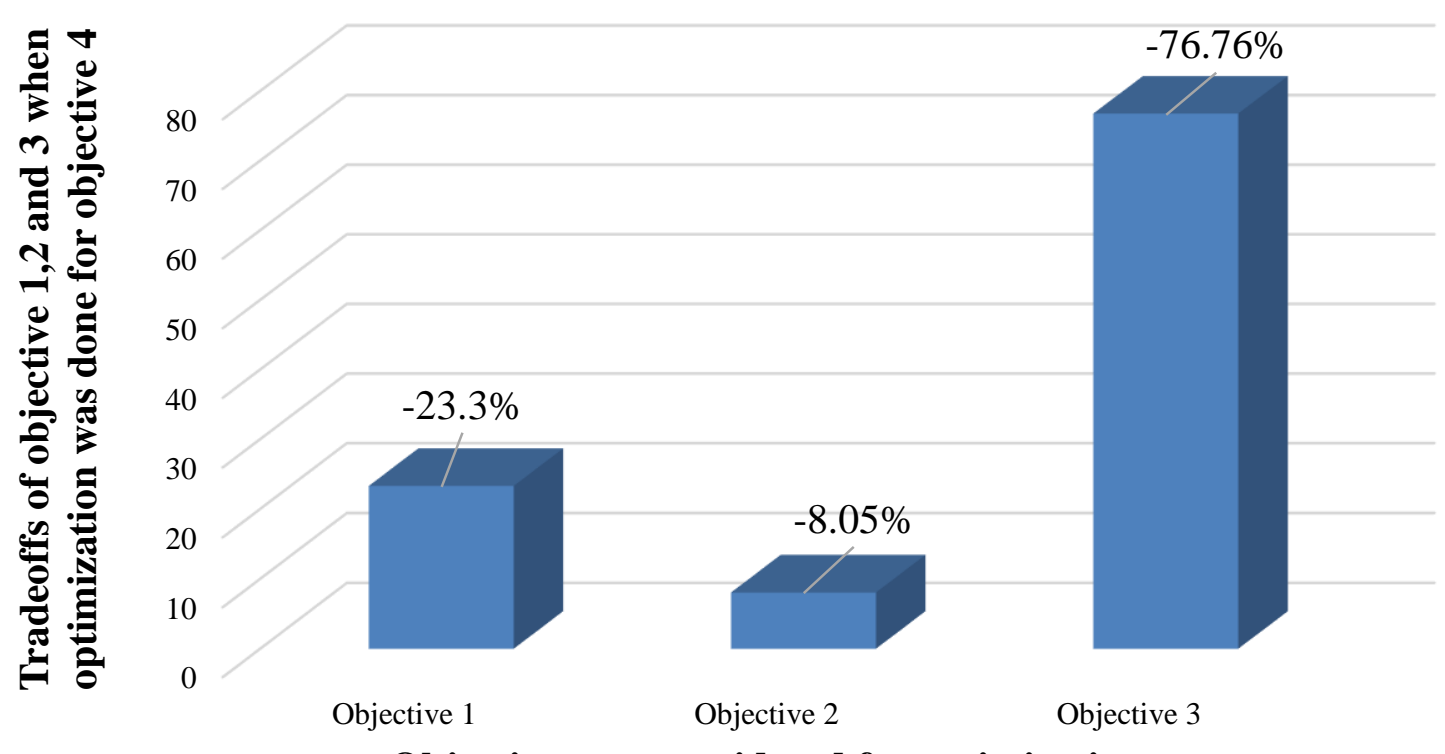

Objectives not considered for optimization

Figure 13 Tradeoff in terms of Three Objectives When Drivers' and Passengers' Matching Was Optimized for System-wide Drivers' Profit

\subsubsection{Minimization of system-wide passengers' waiting time as the matching objective}

Optimization of the ride sharing matching service considering minimization of system-wide passengers' waiting time (objective 1) increased system-wide detour distance by $40.4 \%$ for the example ridesharing scenario (Figure 10). Though optimizing the service to minimize waiting time is favorable to passengers as they need to wait less time at pick-up locations, drivers could feel less motivated as they need to do longer detours to serve passengers. In addition, drivers had to incur $7.47 \%$ less profit compared to the best profit level in this ridesharing scenario for the set of generated routes and four drivers' and riders' matching objectives. Compensating drivers in terms of additional profits and on-board passengers in terms of lower trip cost for longer detours could be an option to encourage longer detours for passenger pick-ups and drop-offs. A detour based pricing scheme can be considered in this regard which will improve both drivers' and on board passengers' service satisfaction level. It will incentivize detouring, increase profit for drivers, save travel cost for passengers and at the same time minimize passengers' waiting time at pick-up point under this objective. However, as optimization of ridesharing drivers' and riders' matching for this matching objective increased system-wide VMT by only $2.09 \%$ from the lowest 
VMT value found from four matching objectives' optimization for the set of generated routes, selection of this objective can ensure nearly minimum use of fuel and transportation infrastructure.

\subsubsection{Minimization of system-wide VMT as the matching objective}

System-wide VMT minimization (Objective 2) performed relatively well (Figure 11) as tradeoffs for other three objectives were relatively low. The solution found from optimization of this objective generated $11 \%$ more system-wide passengers' waiting time, $21.21 \%$ more system- wide detour distance which was the least possible tradeoff from the respective best result found for the set of generated routes and all four matching optimization objectives. Optimizing the ridesharing service for minimum system-wide VMT reduced drivers' profit by $8.67 \%$ as drivers were not allowed to pick-up more passengers by additional/longer detours which could increase systemwide VMT. A detour based pricing scheme can reduce tradeoff related to drivers' profit and at the same time incentivize detouring among drivers and passengers (Biswas et al., 2017).

\subsubsection{Minimization of system-wide detour distance as the matching objective}

System-wide detour distance minimization (objective 3) increased system-wide VMT by $3.58 \%$ compared to the resulted lowest VMT (i.e., optimized for minimization of VMT) for the set of generated routes (Figure 12). In this optimization scenario, system-wide waiting time of passengers (objective 1) was increased by $19.91 \%$ and system-wide drivers' profit (objective 4) was reduced by $21.27 \%$ from their respective best value found in optimization of four matching objectives for the set of generated routes. In this scenario, ride requests were served which could fulfill by minimizing detour distance through minimum deviation from the shortest path between drivers' origin and destination. Though minimization of detour distance objective did not increase VMT much from the lowest VMT level determined, minimum number of requests could be served (shown in Figure 6) and the passengers of unassigned requests have to look for alternative travel options other than ridesharing. Moreover, minimization of detour distance objective reduced drivers' profit by $21.27 \%$ from the best profit level for the set of generated routes considering all four matching objectives. As there were less passengers sharing ride due to minimum detour, drivers' profit level was reduced. Applying this ride matching objective could demotivate drivers due to lower monetary incentive. Moreover, matching agency or government transportation agency usually want ridesharing among maximum number of users to reduce personal car dependency 
thereby serve more travel demand in fewer vehicles, but this ridesharing matching objective failed to serve the overall demand compared to other three matching objectives.

\subsubsection{Maximization of system-wide drivers' profit as the matching objective}

Performances of the other three objectives are relatively poor under the system-wide drivers' profit maximization (objective 4) scenario. The possible reason is that in order to maximize profit, drivers need to travel more detour distance to serve more passengers which increase system-wide VMT. When profit maximization objective was applied, system-wide VMT and detour distance had increased by $8.05 \%$ and $76.76 \%$ respectively from their respective lowest values found in evaluation (Figure 13). As a single driver needed to accommodate multiple passengers' requests, overall passengers' waiting time also increased. In this case, overall system-wide passengers need to wait 145.5 minutes, which was $23.3 \%$ higher than the lowest waiting time scenario for the set

of generated routes and all four matching optimization objectives. From the perspective of government transportation agency, this matching objective increased system-wide VMT which could increase congestions in already congested cities. However, selection of drivers' profit maximization objective for drivers and riders matching minimized passengers' travel cost (Figure 7) as more passengers shared the trip cost, and could act as monetary incentive for passengers who will continue ridesharing mode at the expense of higher system-wide VMT, detour distance and waiting time. 


\section{CHAPTER 5: CONCLUSIONS AND FUTURE RESEARCH DIRECTIONS}

The chapter is divided into two subsections. The first section summarizes the research conclusions and the second section presents future research directions.

\subsection{Conclusions}

Ridesharing services have a strong potential to improve the performance of the transportation system by matching drivers and passengers with less number of vehicles. The most common challenge in developing an efficient ridesharing service system is the selection of appropriate objective(s) to match drivers and riders in order to achieve certain system-level performance. The matching of drivers and riders based on different objectives reflecting multiple stakeholders' concerns have shown different system performance efficiencies. In this study ridesharing drivers and riders matching were performed considering four different matching optimization objectives. The selected four objectives are: (i) minimization of system-wide waiting time of passengers (ii) minimization of system-wide VMT (iii) minimization of system-wide detour distance, and (iv) maximization of system-wide drivers' profit. This thesis evaluated the relative effectiveness of those matching optimization objectives and tradeoffs of considering only one objective over other objectives. This tradeoff analysis demonstrated the effectiveness and deficiencies of four objectives in providing ridesharing in a hypothetical ridesharing scenario.

Minimization of system-wide waiting time and minimization of system-wide VMT objectives showed maximum number of passenger handling capacity (75\%). In the contrary system-wide detour distance minimization objective served the minimum number of passengers $(67 \%)$ because of the minimum detour flexibility of this objective that prohibits significant deviation from original route to serve more riders.

Maximization of system-wide drivers' profit increased the travel cost savings of riders by generating lower trip cost compared to direct taxi service. To maximize profit, drivers needed to share rides with the maximum number of passengers, and riders got the opportunity to cut off trip costs. System-wide waiting time minimization as matching objective offered the least travel cost savings to riders. This objective discouraged drivers to share rides to those passengers who need significant amount of time to pick-up, which led to lower number of passengers to share total trip cost. 
Two trip efficiency indices, waiting time index and ride time index were calculated to compare system-level performance of the four objectives. System-wide waiting time minimization as matching optimization objective provided the highest value in terms of waiting time index. System-wide detour distance minimization reflected the best performance in terms of ride time index.

Tradeoff evaluation of objectives revealed that system-wide VMT minimization objective performed best with least sacrifices on the other three objectives from their respective best performance level for the set of generated routes considering all four matching optimization objectives. System-wide drivers' profit maximization objective imposed the highest sacrifices on the other three objectives from their respective best performance level for the set of generated routes considering all four matching optimization objectives, though this objective provided highest incentives for drivers and riders in terms of maximizing profit and travel cost savings respectively. System-wide minimization of detour distance was found to be the least flexible in providing shared rides.

Findings of this research will help researchers and practitioners to develop ridesharing services considering multiple matching objectives, and choose the best matching objective considering relative effectiveness and tradeoffs in terms of other objectives for a particular ridesharing setting. 


\subsection{Future Research Directions}

While, several past studies considered multiple objectives in developing riders and drivers matching algorithms, no study explored the relative tradeoffs of implementing one objective and associated tradeoffs of other objectives. In this section, several future research directions are identified that could provide even better insights in developing ridesharing services.

- Travel time minimization considering traffic congestion is an important objective for both drivers and passengers which was not considered in this study and can be included in future studies. System-wide users travel time minimization as a matching objective will capture real time traffic scenarios such as congestion delay, delay due to non-recurrent scenarios.

- A hypothetical transportation network and ridesharing scenario was used in this research. Inclusion of real transportation networks and ridesharing scenario will provide the validity of proposed ridesharing matching models.

- Trip characteristics (e.g., trip time and waiting time flexibility) vary with trip purposes (e.g., shopping trip, work trip). Future studies should explore the applicability of proposed matching objectives in satisfying different types of trips.

- This study only considered ridesharing mode during drivers and riders matching. But in the real world there exists several modes for making trips. Besides integrated multimodal service can generate more system-level benefits. For this reason, effectiveness of matching objectives should be tested in integrated multimodal scenarios. 


\section{REFERENCES}

1. Giuliano, G., \& M. Wachs. Responding to Congestion and Traffic Growth: Transportation Demand Management. University of California Transportation Center, University of California, Berkeley, CA, 1992.

2. Hanks Jr, J. W., and T. J. Lomax. Roadway congestion in major urban areas: 1982 to 1988. Transportation Research Record, 1991. Issue 1305. 177-189.

3. Meyer, M. D. Demand management as an element of transportation policy: using carrots and sticks to influence travel behavior. Transportation Research Part A: Policy and Practice, 1999.

4. Traffic Gridlock Sets New Records for Traveler Misery. 2015. https://mobility.tamu.edu/ums/media-information/press-release/. Accessed June 2018.

5. Are we Reaching the End of the Single-Occupancy Vehicle? 2015. https://blog.cargurus.com/2015/12/09/are-we-reaching-the-end-of-the-singleoccupancy-vehicle. Accessed April 2018.

6. Jung, J., R. Jayakrishnan, \& J. Y. Park. Design and modeling of real-time shared-taxi dispatch algorithms. Transportation Research Board 92nd Annual Meeting, 2013. Paper No 13-1798

7. Cohen, B., \& J. Kietzmann. Ride on! Mobility business models for the sharing economy. Organization \& Environment, 2014. 27(3): 279-296.

8. Amey, A., J. Attanucci, and R. Mishalani. Real-time ridesharing: opportunities and challenges in using mobile phone technology to improve rideshare services. Transportation Research Record: Journal of the Transportation Research Board, 2011. 2217: 103-110.

9. Beirão, G., \& J. S. Cabral. Understanding attitudes towards public transport and private car: A qualitative study. Transport policy,2007. 14(6): 478-489.

10. Ge Y., H. Xiong, A. Tuzhilin, K. Xiao, M. Gruteser, and M. Pazzani. An energy- efficient mobile recommender system. 16th ACM SIGKDD international conference on Knowledge discovery and data mining, 2010. 899-908.

11. Hou Y., X. Li, Y. Zhao, X. Jia, A.W. Sadek, K. Hulme, C. Qiao. Towards efficient vacant taxis cruising guidance, Global Communications Conference, 2013. 54-59. 
12. Qu M., H. Zhu, J. Liu, G. Liu, and H. Xiong. A cost-effective recommender system for taxi drivers. Proceedings of the 20th ACM SIGKDD international conference on Knowledge discovery and data mining, 2014. 45-54.

13. Hsueh, Y. L., R. H Hwang, and Y. T. Chen. An effective taxi recommender system based on a spatiotemporal factor analysis model. Computing, Networking and Communications (ICNC), International Conference, IEEE, 2014. 429-433.

14. Herbawi, W., \& M. Weber. The ridematching problem with time windows in dynamic ridesharing: A model and a genetic algorithm. Evolutionary Computation (CEC), IEEE, 2012. $1-8$

15. Armant, V., \& K. N. Brown. Minimizing the driving distance in ride sharing systems. Tools with Artificial Intelligence (ICTAI), IEEE 26th International Conference, 2014. $568-575$.

16. Ehsani, P., \& J. Y. Yu. The merits of sharing a ride. Communication, Control, and Computing (Allerton), 55th Annual Allerton Conference, IEEE, 2017. 776-782.

17. Biswas, A., R. Gopalakrishnan, T. Tulabandhula, A. Metrewar, K. Mukherjee, and R. S. Thangaraj. Impact of Detour-Aware Policies on Maximizing Profit in Ridesharing. 2017. arXiv preprint arXiv:1706.02682.

18. Lin, Y., W. Li, F. Qiu, \& H. Xu. Research on optimization of vehicle routing problem for ride-sharing taxi. Procedia-Social and Behavioral Sciences, 2012. 43: 494-502.

19. Herbawi, W., \& M. Weber. Ant colony vs. genetic multiobjective route planning in dynamic multi-hop ridesharing. Tools with Artificial Intelligence (ICTAI), 23rd IEEE International Conference, November 2011. 282-288.

20. Herbawi, W., and M. Weber. Comparison of multiobjective evolutionary algorithms for solving the multiobjective route planning in dynamic multi-hop ridesharing. Evolutionary Computation (CEC), IEEE, June 2011. 2099-2106.

21. Agatz, N. A., A. L. Erera, M. W. Savelsbergh \& X. Wang. Dynamic ride-sharing: A simulation study in metro Atlanta. Transportation Research Part B: Methodological, 2011. 45(9): 1450-1464.

22. Wang, X., N. Agatz, \& A. Erera. Stable matching for dynamic ride-sharing systems. Transportation Science, 2017. https://doi.org/10.1287/trsc.2017.0768. 
23. Stiglic, M., N. Agatz, M. Savelsbergh, \& M. Gradisar. The benefits of meeting points in ride-sharing systems. Transportation Research Part B: Methodological, 2015. 82: 36-53.

24. Li, X., S. Hu, W. Fan, and K. Deng. Modeling an enhanced ridesharing system with meet points and time windows. PloS one, 2018. 13(5).

25. Kaan, L., and E. V. Olinick. The vanpool assignment problem: Optimization models and solution algorithms. Computers \& Industrial Engineering, 2013. 66(1): 24-40.

26. Ma, S., Y. Zheng, Y., \& O. Wolfson. T-share: A large-scale dynamic taxi ridesharing service. Data Engineering (ICDE), 29th International Conference, IEEE, 2013. 410-421.

27. Litman T. Public Transportation Impact on Rural and Small Towns. American Public Transportation Association, 2017. https://www.apta.com/resources/reportsandpublications/Documents/APTA-RuralTransit-2017.pdf. Accessed June 2018.

28. LeBlanc S. Studies are increasingly clear: Uber and Lyft congest cities. 2018. http://www.chicagotribune.com/bluesky/technology/ct-uber-lyft-congestion-20180225story.html.Accessed May 2018.

29. Bagloee, S. A., M. Tavana, M. Asadi, \& T. Oliver. Autonomous vehicles: challenges, opportunities, and future implications for transportation policies. Journal of Modern Transportation,2016. 24(4): 284-303.

30. Piotrowski M. Vehicle Miles Travelled and Ridesharing. 2017. http://energyfuse.org/vehicle-miles-traveled-ridesharing/. Accessed September 2018.

31. U.S Energy Information Administration. (n.d.). https://www.eia.gov/ .Accessed September 2018 .

32. Hensley R., A. Padhi, and J. Salazar. Cracks in Ridesharing market-and how to fill them. 2017. https://www.mckinsey.com/industries/automotive-and-assembly/ourinsights/cracks-in-the-ridesharing-market-and-how-to-fill-them. Accessed September 2018.

33. Harolds S. Maximize Uber Earnings When Driving. 2018. https://www.ridester.com/maximize-uber-earnings/. Accessed October 22, 2018. 
34. Dustin. Lyft Comes Through on Ridiculous Surge Pricing. 2017. https://runningwithmiles.boardingarea.com/lyft-surge-pricing/. Accessed October 22,2018 . 